Assessment of the National Park network of mainland Spain by the Insecurity Index of vertebrate species

Alba Estrada \& Raimundo Real

Plos One

S1 Appendix. Variables included at each step of the modelling process and final favourability models. Variables included at each step of the modelling process (i.e., pairwise correlations, spatial descriptor, FDR, and forward-backward stepwise) for each species. X: longitude; Y:

latitude. Variables selected for the spatial descriptor were those selected in the trend surface approach (spatial model). The variables included in the forward-backward stepwise are those of the final favourability models. Variables are sorted according to their order of entrance in the models. $\beta$ : coefficients; SE: standard errors; Sig: significance: ${ }^{* * *}<0.001, * *<0.01,{ }^{*}<0.05$, ns>0.05. Evaluation metrics of the models are also shown. Variable codes as in S1 Table. "Spatial" in final favourability models is the linear combination of selected spatial variables with their coefficients and the intercept of the spatial model (see Estrada et al. 2016 Plos One). 


\section{Chioglossa lusitanica}

Pairwise correlations, spatial descriptor and FDR

Human, lithology and land use variables were not correlated, thus all of them were selected in pairwise correlations.

Variables selected

\begin{tabular}{|c|c|c|c|}
\hline Climate & Topography & Spatial & Variables excluded by the FDR \\
\hline DP1Win & Alt & $\mathrm{XY}$ & $\mathrm{HPd}$ \\
\hline DP30Spr & SE & $x^{2}$ & DTnOSum \\
\hline DP30Sum & WE & $X^{2} Y$ & Shrub \\
\hline DTnOWin & CTI & $X Y^{2}$ & \\
\hline DTnOSum & & & \\
\hline DTn20 & & & \\
\hline DTx25Spr & & & \\
\hline TRan & & & \\
\hline SIDAut & & & \\
\hline AET & & & \\
\hline
\end{tabular}

Final favourability model

\begin{tabular}{lrrlll}
\multicolumn{1}{c}{ Variables } & B & SE & Sig & Evaluation metrics & \\
(Intercept) & 14.104053 & 4.332457 & $* *$ & AUC & 0.9853935 \\
Spatial & 0.75198 & 0.196765 & $* * *$ & Kappa & 0.4657085 \\
Sil & 4.340705 & 0.923729 & $* * *$ & Sensitivity & 0.9880952 \\
DP30Spr & 0.236318 & 0.135775 & $\mathrm{~ns}$ & Specificity & 0.9334630 \\
DTnOWin & -0.042055 & 0.010895 & $* * *$ & CCR & 0.935192 \\
CTI & -0.906338 & 0.251222 & $* * *$ & HL & $11.3658311 \mathrm{~ns}$ \\
DHi & -0.015947 & 0.007593 & $*$ & & \\
DTn20 & -0.435574 & 0.193528 & $*$ & & \\
Clay & 3.87317 & 1.439383 & $* *$ & & \\
Calc & 2.86569 & 1.209104 & $*$ & & \\
BLF & -1.552821 & 0.887971 & $\mathrm{~ns}$ & & \\
Grav & 4.034756 & 2.281961 & $\mathrm{~ns}$ & & \\
SE & -0.016587 & 0.010997 & $\mathrm{~ns}$ & &
\end{tabular}




\section{Euproctus asper}

Pairwise correlations, spatial descriptor and FDR

Human, lithology and land use variables were not correlated, thus all of them were selected in pairwise correlations.

Variables selected

$\begin{array}{llll}\text { Climate } & \text { Topography } & \text { Spatial } & \text { Variables excluded by the FDR } \\ \text { DP10Spr Alt } & X & \text { Shrub } \\ \text { DP10Sum SE } & X Y & \text { WE } \\ \text { DTnOWin WE } & X^{2} & \text { Gyps } \\ \text { DTn0Sum CTI } & X^{2} Y & \\ \text { DTn20 } & X^{2} & \\ \text { TRan } & & \\ \text { AET } & & \end{array}$

Final favourability model

\begin{tabular}{lrrlll}
\multicolumn{1}{c}{ Variables } & B & SE & Sig & Evaluation metrics \\
(Intercept) & -2.965059 & 2.667609 & $\mathrm{~ns}$ & AUC & 0.9960155 \\
Spatial & 1.481486 & 0.222381 & $* * *$ & Kappa & 0.7169263 \\
BLF & 3.721701 & 0.98703 & $* * *$ & Sensitivity & 1 \\
HPd & -0.024717 & 0.014962 & $\mathrm{~ns}$ & Specificity & 0.9726509 \\
DTn20 & -0.189029 & 0.045073 & $* * *$ & CCR & 0.9736247 \\
U500 & -0.018825 & 0.007658 & $*$ & HL & $22.6335351 * *$ \\
NIAL & -7.78196 & 2.232869 & $* * *$ & & \\
Past & -7.26826 & 2.898036 & $*$ & & \\
MF & 2.914323 & 1.988447 & $\mathrm{~ns}$ & & \\
Alt & 0.003819 & 0.001015 & $* * *$ & & \\
DTnOWin & -0.105248 & 0.028221 & $* * *$ & & \\
TRan & 0.542185 & 0.189932 & $* *$ & & \\
DTnOSum & -0.625408 & 0.407322 & $\mathrm{~ns}$ & &
\end{tabular}




\section{Pleurodeles waltI}

Pairwise correlations, spatial descriptor and FDR

Human, lithology and land use variables were not correlated, thus all of them were selected in pairwise correlations.

\section{Variables selected}

$\begin{array}{llll}\text { Climate } & \text { Topography } & \text { Spatial } & \text { Variables excluded by the FDR } \\ \text { DP10Spr } & \text { Alt } & Y & \text { Shrub } \\ \text { DTnOSum } & \text { DAlt } & X & \text { HPd } \\ \text { DTn20 } & \text { SE } & X Y & \text { SE } \\ \text { TRan } & \text { WE } & X^{2} & \text { BLF } \\ \text { TXAut } & \text { CTI } & X^{3} & \text { Clay } \\ \text { SIDSum } & & Y^{2} & \\ \text { SISWin } & & Y^{3} & \\ \text { AET } & & X^{2} Y & \\ & & X^{2} & \end{array}$

Final favourability model

$\begin{array}{lrrlll}\text { Variables } & \text { B } & \text { SE } & \text { Sig } & \text { Evaluation metrics } \\ \text { (Intercept) } & 3.97 \mathrm{E}+00 & 1.45 \mathrm{E}+00 & * * & \text { AUC } & 0.8575984 \\ \text { Spatial } & 1.06 \mathrm{E}+00 & 6.04 \mathrm{E}-02 & * * * & \text { Kappa } & 0.4343372 \\ \text { DTn0Sum } & -4.15 \mathrm{E}+00 & 6.50 \mathrm{E}-01 & * * * & \text { Sensitivity } & 0.8536386 \\ \text { Alt } & 6.67 \mathrm{E}-04 & 1.59 \mathrm{E}-04 & * * * & \text { Specificity } & 0.7072215 \\ \text { U500 } & 2.44 \mathrm{E}-03 & 7.90 \mathrm{E}-04 & * * & \text { CCR } & 0.7409570 \\ \text { Gyps } & -1.52 \mathrm{E}+01 & 1.13 \mathrm{E}+01 & \mathrm{~ns} & \mathrm{HL} & 50.1243829 * * * \\ \text { CTI } & -1.59 \mathrm{E}-01 & 6.07 \mathrm{E}-02 & * * & & \\ \text { Grav } & 6.01 \mathrm{E}-01 & 2.33 \mathrm{E}-01 & * * & & \\ \text { DP10Spr } & -1.39 \mathrm{E}-01 & 4.20 \mathrm{E}-02 & * * * & & \\ \text { AET } & 8.82 \mathrm{E}-04 & 4.52 \mathrm{E}-04 & \mathrm{~ns} & & \\ \text { Sil } & 2.73 \mathrm{E}-01 & 1.31 \mathrm{E}-01 & * & & \\ \text { SIDSum } & -2.94 \mathrm{E}-01 & 1.53 \mathrm{E}-01 & \mathrm{~ns} & & \end{array}$




\section{Bufo calamita}

Pairwise correlations, spatial descriptor and FDR

Human, lithology and land use variables were not correlated, thus all of them were selected in pairwise correlations.

Variables selected

$\begin{array}{llll}\text { Climate } & \text { Topography } & \text { Spatial } & \text { Variables excluded by the FDR } \\ \text { DP1 } & \text { Alt } & Y & \text { DTx25Spr } \\ \text { DP30Aut } & \text { Slop } & X & \text { WE } \\ \text { DP30Sum } & \text { SE } & X Y & \text { Shrub } \\ \text { DTn0Sum } & \text { WE } & X^{2} & \text { DHi } \\ \text { DTn20 } & X^{3} & \text { Calc } \\ \text { DTx25Spr } & Y^{2} & \text { Gyps } \\ \text { TRan } & Y^{3} & \text { Alt } \\ \text { TnJul } & X^{2} & \\ \text { AET } & & \end{array}$

Final favourability model

\begin{tabular}{lrrlll}
$\begin{array}{lrll}\text { Variables } \\
\text { (Intercept) }\end{array}$ & \multicolumn{1}{c}{ B } & SE & Sig & Evaluation metrics & \\
Spatial & $7.69 \mathrm{E}-01$ & $7.40 \mathrm{E}-02$ & $* * *$ & Kappa & 0.6886401 \\
U100 & $-4.69 \mathrm{E}-03$ & $1.13 \mathrm{E}-03$ & $* * *$ & Sensitivity & 0.2716559 \\
Past & $-5.61 \mathrm{E}+00$ & $9.39 \mathrm{E}-01$ & $* * *$ & Specificity & 0.6611627 \\
HPd & $8.78 \mathrm{E}-04$ & $2.05 \mathrm{E}-04$ & $* * *$ & CCR & 0.6384702 \\
CF & $1.10 \mathrm{E}+00$ & $2.44 \mathrm{E}-01$ & $* * *$ & $\mathrm{HL}$ & $40.4083661 * * *$ \\
BLF & $7.82 \mathrm{E}-01$ & $2.79 \mathrm{E}-01$ & $* *$ & & \\
DP30Aut & $-3.18 \mathrm{E}-01$ & $4.30 \mathrm{E}-02$ & $* * *$ & & \\
Sil & $5.15 \mathrm{E}-01$ & $8.99 \mathrm{E}-02$ & $* * *$ & & \\
U500 & $-3.52 \mathrm{E}-03$ & $6.07 \mathrm{E}-04$ & $* * *$ & & \\
TRan & $-5.64 \mathrm{E}-02$ & $1.81 \mathrm{E}-02$ & $* *$ & & \\
AET & $7.33 \mathrm{E}-04$ & $2.90 \mathrm{E}-04$ & $*$ & & \\
SE & 0.0034787 & 0.0022651 & $\mathrm{~ns}$ & &
\end{tabular}




\section{Hyla meridionalis}

Pairwise correlations, spatial descriptor and FDR

Human, lithology and land use variables were not correlated, thus all of them were selected in pairwise correlations.

\section{Variables selected}

$\begin{array}{llll}\text { Climate } & \text { Topography } & \text { Spatial } & \text { Variables excluded by the FDR } \\ \text { DP30Win } & \text { Alt } & Y & \text { CF } \\ \text { DP30Sum } & \text { DAlt } & X Y & \text { Gyps } \\ \text { DTnOSum } & \text { SE } & X^{3} & \text { TRan } \\ \text { DTn20 } & \text { WE } & Y^{2} & \text { Shrub } \\ \text { TAut } & \text { CTI } & Y^{3} & \text { DHi } \\ \text { TRan } & & X^{2} Y & \\ \text { ROff } & & X^{2} & \\ \text { AET } & & & \end{array}$

Final favourability model

\begin{tabular}{lrrlll} 
Variables & \multicolumn{1}{c}{ B } & SE & Sig & Evaluation metrics \\
(Intercept) & $1.91 \mathrm{E}+00$ & $1.99 \mathrm{E}+00$ & $\mathrm{~ns}$ & AUC & 0.9494523 \\
Spatial & $8.52 \mathrm{E}-01$ & $4.14 \mathrm{E}-02$ & $* * *$ & Kappa & 0.5852454 \\
AET & $2.52 \mathrm{E}-03$ & $5.07 \mathrm{E}-04$ & $* * *$ & Sensitivity & 0.9322251 \\
DTn20 & $1.85 \mathrm{E}-02$ & $4.88 \mathrm{E}-03$ & $* * *$ & Specificity & 0.8486522 \\
Sil & $-1.16 \mathrm{E}+00$ & $4.46 \mathrm{E}-01$ & $* *$ & CCR & 0.8609646 \\
SE & $1.59 \mathrm{E}-02$ & $4.68 \mathrm{E}-03$ & $* * *$ & $\mathrm{HL}$ & $56.7003716 * * *$ \\
Alt & $-1.09 \mathrm{E}-03$ & $2.80 \mathrm{E}-04$ & $* * *$ & & \\
CTI & $-2.40 \mathrm{E}-01$ & $8.81 \mathrm{E}-02$ & $* *$ & & \\
HPd & $3.84 \mathrm{E}-04$ & $1.35 \mathrm{E}-04$ & $* *$ & & \\
Past & $5.48 \mathrm{E}+00$ & $2.15 \mathrm{E}+00$ & $*$ & & \\
Clay & $-2.06 \mathrm{E}+00$ & $4.67 \mathrm{E}-01$ & $* * *$ & & \\
WE & $8.26 \mathrm{E}-03$ & $5.75 \mathrm{E}-03$ & $\mathrm{~ns}$ & & \\
BLF & 1.2950793 & 0.6347605 & & & \\
Grav & -1.9231928 & 0.5357702 & $* * *$ & & \\
Calc & -1.7108759 & 0.4807249 & $* * *$ & &
\end{tabular}




\section{Rana iberica}

Pairwise correlations, spatial descriptor and FDR

Human, lithology and land use variables were not correlated, thus all of them were selected in pairwise correlations.

Variables selected

$\begin{array}{llll}\text { Climate } & \text { Topography } & \text { Spatial } & \text { Variables excluded by the FDR } \\ \text { DP10Win } & \text { Alt } & Y & \text { Alt } \\ \text { DP30Spr } & \text { DAlt } & X & \text { SE } \\ \text { DP30Sum SE } & X Y & \text { HPd } \\ \text { DTnOSum } & \text { WE } & X^{2} & \\ \text { DTn20 CTI } & X^{3} & \\ \text { TRan } & Y^{2} & \\ \text { SISWin } & Y^{3} & \\ \text { PET } & X^{2} Y & \\ \text { AET } & X Y^{2} & \end{array}$

Final favourability model

\begin{tabular}{lrrlll} 
Variables & \multicolumn{1}{c}{ B } & \multicolumn{1}{c}{ SE } & Sig & Evaluation metrics & \\
(Intercept) & $-6.66 \mathrm{E}+00$ & $1.80 \mathrm{E}+00$ & $* * *$ & AUC & 0.9834648 \\
Spatial & $1.04 \mathrm{E}+00$ & $1.08 \mathrm{E}-01$ & $* * *$ & Kappa & 0.6569974 \\
DAlt & $1.20 \mathrm{E}-03$ & $3.16 \mathrm{E}-04$ & $* * *$ & Sensitivity & 0.9522822 \\
Sil & $5.92 \mathrm{E}+00$ & $8.51 \mathrm{E}-01$ & $* * *$ & Specificity & 0.9218815 \\
U500 & $-9.78 \mathrm{E}-03$ & $1.72 \mathrm{E}-03$ & $* * *$ & CCR & 0.9246420 \\
CF & $4.13 \mathrm{E}+00$ & $8.44 \mathrm{E}-01$ & $* * *$ & HL & $20.9191192 * *$ \\
PET & $-1.75 \mathrm{E}-02$ & $2.99 \mathrm{E}-03$ & $* * *$ & & \\
AET & $1.08 \mathrm{E}-02$ & $2.29 \mathrm{E}-03$ & $* * *$ & & \\
DP30Sum & $-1.33 \mathrm{E}+00$ & $3.23 \mathrm{E}-01$ & $* * *$ & & \\
Calc & $5.80 \mathrm{E}+00$ & $9.66 \mathrm{E}-01$ & $* * *$ & & \\
Grav & $6.10 \mathrm{E}+00$ & $1.19 \mathrm{E}+00$ & $* * *$ & & \\
DP10Win & $1.09 \mathrm{E}-01$ & $3.31 \mathrm{E}-02$ & $* *$ & & \\
Clay & 3.7404198 & 1.0882839 & $* * *$ & & \\
SISWin & 4.8799604 & 0.983512 & $* * *$ & & \\
TRan & -0.2966148 & 0.0723339 & $* * *$ & & \\
DTn0Sum & 0.5978934 & 0.259484 & $*$ & & \\
MF & 2.5633932 & 0.8419069 & $* *$ & & \\
BLF & 2.1377256 & 0.7343627 & $* *$ & & \\
Shrub & 1.2962815 & 0.5504615 & $*$ & &
\end{tabular}




\section{Rana pyrenaica}

Pairwise correlations, spatial descriptor and FDR

Human, lithology and land use variables were not correlated, thus all of them were selected in pairwise correlations.

Variables selected

$\begin{array}{llll}\text { Climate } & \text { Topography } & \text { Spatial } & \text { Variables excluded by the FDR } \\ \text { DP10Spr } & \text { Alt } & X & \text { WE } \\ \text { DP10Sum } & \text { SE } & X Y & \text { Gyps } \\ \text { DTn0Sum } & \text { WE } & X^{3} & \text { BLF } \\ \text { DTn20 CTI } & Y^{2} & \text { Past } \\ \text { TRan } & Y^{3} & \text { Sil } \\ \text { TXAut } & X^{2} Y & \text { U500 } \\ \text { AET } & X Y^{2} & \end{array}$

Final favourability model

\begin{tabular}{lcclll}
\multicolumn{1}{c}{ Variables } & B & SE & Sig & & Evaluation metrics \\
(Intercept) & $3.31 \mathrm{E}+02$ & $1.09 \mathrm{E}+02$ & $* *$ & AUC & 1 \\
Spatial & $4.05 \mathrm{E}+01$ & $1.29 \mathrm{E}+01$ & $* *$ & Kappa & 0.9051913 \\
Calc & $1.23 \mathrm{E}+02$ & $3.98 \mathrm{E}+01$ & $* *$ & Sensitivity & 1 \\
CF & $4.07 \mathrm{E}+02$ & $1.31 \mathrm{E}+02$ & $* *$ & Specificity & 0.9990537 \\
Shrub & $-2.49 \mathrm{E}+02$ & $8.74 \mathrm{E}+01$ & $* *$ & CCR & 0.9990580 \\
Clay & $3.68 \mathrm{E}+02$ & $1.19 \mathrm{E}+02$ & $* *$ & HL & $0.9031554 \mathrm{~ns}$ \\
DHi & $-1.78 \mathrm{E}+00$ & $6.93 \mathrm{E}-01$ & $*$ & & \\
TRan & $-2.24 \mathrm{E}+01$ & $7.59 \mathrm{E}+00$ & $* *$ & & \\
MF & $4.22 \mathrm{E}+01$ & $1.84 \mathrm{E}+01$ & $*$ & &
\end{tabular}




\section{Emys orbicularis}

Pairwise correlations, spatial descriptor and FDR

Human, lithology and land use variables were not correlated, thus all of them were selected in pairwise correlations.

Variables selected

$\begin{array}{llll}\text { Climate } & \text { Topography } & \text { Spatial } & \text { Variables excluded by the FDR } \\ \text { DP10Sum } & \text { Alt } & Y & \text { Grav } \\ \text { DP30Spr } & \text { DAlt } & X & \text { Gyps } \\ \text { DTnOSum } & \text { SE } & X Y & \text { U100 } \\ \text { DTn20 } & \text { WE } & X^{3} & \text { WE } \\ \text { TRan } & \text { CTI } & Y^{2} & \\ \text { TXAut } & & Y^{3} & \\ \text { ROff } & X^{2} Y & \\ \text { AET } & X Y^{2} & \end{array}$

Final favourability model

\begin{tabular}{lrrlll}
$\begin{array}{lrrr}\text { Variables } \\
\text { (Intercept) }\end{array}$ & \multicolumn{1}{c}{ B } & \multicolumn{1}{c}{ SE } & Sig & Evaluation metrics & \\
Spatial & $5.16 \mathrm{E}-01$ & $2.08 \mathrm{E}+00$ & $\mathrm{~ns}$ & AUC & 0.8112590 \\
NIAL & $-6.23 \mathrm{E}-01$ & $3.10 \mathrm{E}-02$ & $* *$ & Kappa & 0.2116908 \\
DTn0Sum & $-1.75 \mathrm{E}+00$ & $6.98 \mathrm{E}-01$ & $*$ & Sensitivity & 0.7651007 \\
Calc & $-8.82 \mathrm{E}-01$ & $2.20 \mathrm{E}-01$ & $* * *$ & CCR & 0.7183707 \\
DHi & $1.49 \mathrm{E}-02$ & $2.70 \mathrm{E}-03$ & $* * *$ & $\mathrm{HL}$ & 0.7223060 \\
U500 & $-4.85 \mathrm{E}-03$ & $1.07 \mathrm{E}-03$ & $* * *$ & & $20.8598058 * *$ \\
BLF & $2.61 \mathrm{E}+00$ & $4.85 \mathrm{E}-01$ & $* * *$ & & \\
DP10Sum & $-3.56 \mathrm{E}-01$ & $7.44 \mathrm{E}-02$ & $* * *$ & & \\
Clay & $-5.80 \mathrm{E}-01$ & $2.44 \mathrm{E}-01$ & $*$ & & \\
SE & $1.22 \mathrm{E}-02$ & $4.37 \mathrm{E}-03$ & $* *$ & & \\
CTI & $2.44 \mathrm{E}-01$ & $6.91 \mathrm{E}-02$ & $* * *$ & & \\
Shrub & 1.1467561 & 0.3312632 & $* * *$ & & \\
TxAut & -0.2690325 & 0.0569823 & $* * *$ & & \\
Alt & -0.0017543 & 0.0003831 & $* * *$ & & \\
TRan & 0.0802111 & 0.033057 & $*$ & & \\
CF & 1.1775007 & $0.5300833 *$ & &
\end{tabular}




\section{Mauremys leprosa}

Pairwise correlations, spatial descriptor and FDR

Human, lithology and land use variables were not correlated, thus all of them were selected in pairwise correlations.

Variables selected

$\begin{array}{llll}\text { Climate } & \text { Topography } & \text { Spatial } & \text { Variables excluded by the FDR } \\ \text { DP30Sum } & \text { Alt } & X & \text { Clay } \\ \text { DTnOSpr } & \text { DAlt } & X Y & \text { Grav } \\ \text { DTnOSum } & \text { SE } & X^{3} & \text { U100 } \\ \text { DTn20 } & \text { WE } & Y^{2} & \\ \text { PSpr } & \text { CTI } & Y^{3} & \\ \text { TJul } & & X^{2} Y & \\ \text { TRan } & & & \\ \text { AET } & & & \end{array}$

Final favourability model

\begin{tabular}{lrrlll} 
Variables & \multicolumn{1}{c}{ B } & \multicolumn{1}{c}{ SE } & Sig & Evaluation metrics & \\
(Intercept) & $-9.14 \mathrm{E}-01$ & $4.15 \mathrm{E}-01$ & $*$ & AUC & 0.8572861 \\
Spatial & $6.38 \mathrm{E}-01$ & $4.25 \mathrm{E}-02$ & $* * *$ & Kappa & 0.5183574 \\
DTn0Spr & $-4.82 \mathrm{E}-02$ & $7.65 \mathrm{E}-03$ & $* * *$ & Sensitivity & 0.8115038 \\
Sil & $5.55 \mathrm{E}-01$ & $1.23 \mathrm{E}-01$ & $* * *$ & Specificity & 0.7743855 \\
U500 & $-5.59 \mathrm{E}-03$ & $7.78 \mathrm{E}-04$ & $* * *$ & CCR & 0.7844763 \\
DTn0Sum & $-2.75 \mathrm{E}+00$ & $6.57 \mathrm{E}-01$ & $* * *$ & $\mathrm{HL}$ & $41.2214238 * * *$ \\
Shrub & $8.07 \mathrm{E}-01$ & $2.27 \mathrm{E}-01$ & $* * *$ & & \\
HPd & $5.89 \mathrm{E}-04$ & $1.40 \mathrm{E}-04$ & $* * *$ & & \\
CF & $1.24 \mathrm{E}+00$ & $3.52 \mathrm{E}-01$ & $* * *$ & & \\
PSpr & $3.26 \mathrm{E}-03$ & $9.92 \mathrm{E}-04$ & $* * *$ & & \\
DP30Sum & $-4.32 \mathrm{E}-01$ & $1.34 \mathrm{E}-01$ & $* *$ & & \\
Past & $-3.68 \mathrm{E}+00$ & $1.77 \mathrm{E}+00$ & $*$ & & \\
DHi & 0.0039865 & $0.001976 *$ & & \\
BLF & 0.8579009 & $0.4324115 *$ & & \\
TRan & 0.0558105 & 0.022311 & & & \\
Gyps & -0.8969753 & 0.5267162 & $\mathrm{~ns}$ & & \\
NIAL & -0.3628105 & 0.2004525 & $\mathrm{~ns}$ & &
\end{tabular}




\section{Testudo graeca}

Pairwise correlations, spatial descriptor and FDR

Human, lithology and land use variables were not correlated, thus all of them were selected in pairwise correlations.

\section{Variables selected}

$\begin{array}{llll}\text { Climate } & \text { Topography } & \text { Spatial } & \text { Variables excluded by the FDR } \\ \text { DP1 } & \text { Alt } & Y & \text { Gyps } \\ \text { DP30Spr } & \text { SE } & X Y & \text { CTI } \\ \text { DTn0Sum } & \text { WE } & X^{2} & \text { Shrub } \\ \text { DTn20 } & \text { CTI } & Y^{2} & \text { CF } \\ \text { DTx25Aut } & Y^{3} & \text { Sil } \\ \text { PSum } & X^{2} Y & \text { Clay } \\ \text { TRan } & X^{2} & \text { HPd } \\ \text { TXWin } & & \text { U100 } \\ \text { AET } & & \end{array}$

Final favourability model

\begin{tabular}{lrrlll}
\multicolumn{1}{c}{ Variables } & B & SE & Sig & Evaluation metrics \\
(Intercept) & $5.87 \mathrm{E}+00$ & $3.57 \mathrm{E}+00$ & $\mathrm{~ns}$ & AUC & 0.9981490 \\
Spatial & $1.00 \mathrm{E}+00$ & $2.15 \mathrm{E}-01$ & $* * *$ & Kappa & 0.4687089 \\
BLF & $-1.92 \mathrm{E}+02$ & $1.14 \mathrm{E}+02$ & $\mathrm{~ns}$ & Sensitivity & 1 \\
WE & $-5.91 \mathrm{E}-02$ & $2.29 \mathrm{E}-02$ & $* *$ & Specificity & 0.9844432 \\
NIAL & $-6.81 \mathrm{E}+00$ & $5.18 \mathrm{E}+00$ & $\mathrm{~ns}$ & $\mathrm{CCR}$ & 0.9845516 \\
DTn20 & $-2.80 \mathrm{E}-02$ & $1.32 \mathrm{E}-02$ & $*$ & $\mathrm{HL}$ & $11.5754054 \mathrm{~ns}$ \\
SE & $-4.06 \mathrm{E}-02$ & $1.86 \mathrm{E}-02$ & $*$ & & \\
DTx25Aut & $1.14 \mathrm{E}-01$ & $5.60 \mathrm{E}-02$ & $*$ & &
\end{tabular}




\section{Anguis fragilis}

Pairwise correlations, spatial descriptor and FDR

Human, lithology and land use variables were not correlated, thus all of them were selected in pairwise correlations.

\section{Variables selected}

$\begin{array}{llll}\text { Climate } & \text { Topography } & \text { Spatial } & \text { Variables excluded by the FDR } \\ \text { DP01 } & \text { Alt } & Y & \text { SE } \\ \text { DP30Spr } & \text { SE } & X & \text { Calc } \\ \text { DP30Sum } & \text { WE } & X^{2} & \text { WE } \\ \text { DTn0Sum } & \text { CTI } & X^{3} & \\ \text { DTn20 } & Y^{2} & \\ \text { TRan } & Y^{3} & \\ \text { TXJul } & X^{2} Y & \\ \text { PET } & X Y^{2} & \\ \text { AET } & & \end{array}$

Final favourability model

\begin{tabular}{|c|c|c|c|c|c|}
\hline Variables & B & SE & Sig & Evaluation metrics & \\
\hline (Intercept) & 2.67E+00 & $1.11 \mathrm{E}+00$ & * & AUC & 0.9391049 \\
\hline Spatial & $6.08 \mathrm{E}-01$ & $4.72 \mathrm{E}-02$ & $* * *$ & Карра & 0.6307209 \\
\hline $\mathrm{AET}$ & 4.05E-03 & $6.73 E-04$ & $* * *$ & Sensitivity & 0.9068136 \\
\hline DP30Sum & 5.89E-01 & 1.49E-01 & $* * *$ & Specificity & 0.8540603 \\
\hline $\mathrm{DHi}$ & $-1.54 \mathrm{E}-02$ & $3.18 \mathrm{E}-03$ & $* * *$ & $\mathrm{CCR}$ & 0.8639789 \\
\hline PET & $-5.92 E-03$ & $1.30 \mathrm{E}-03$ & $* * *$ & $\mathrm{HL}$ & $11.6840720 \mathrm{~ns}$ \\
\hline U500 & $-4.85 E-03$ & $1.11 \mathrm{E}-03$ & $* * *$ & & \\
\hline $\mathrm{HPd}$ & $3.51 \mathrm{E}-04$ & $1.36 \mathrm{E}-04$ & $* *$ & & \\
\hline BLF & $1.47 \mathrm{E}+00$ & $3.92 \mathrm{E}-01$ & $* * *$ & & \\
\hline $\mathrm{CF}$ & $1.63 \mathrm{E}+00$ & 4.18E-01 & $* * *$ & & \\
\hline Past & $-1.67 E+00$ & $6.57 \mathrm{E}-01$ & $*$ & & \\
\hline TRan & $-6.15 E-02$ & $2.91 \mathrm{E}-02$ & $*$ & & \\
\hline DTn20 & 0.0203212 & 0.0081187 & $*$ & & \\
\hline U100 & -0.0040251 & 0.0024434 & ns & & \\
\hline DTnOSum & 0.2843962 & 0.1636721 & ns & & \\
\hline Sil & 0.4647558 & 0.1717781 & $* *$ & & \\
\hline Clay & 0.4879699 & 0.2540485 & ns & & \\
\hline
\end{tabular}




\section{Podarcis muralis}

Pairwise correlations, spatial descriptor and FDR

Human, lithology and land use variables were not correlated, thus all of them were selected in pairwise correlations.

Variables selected

$\begin{array}{llll}\text { Climate } & \text { Topography } & \text { Spatial } & \text { Variables excluded by the FDR } \\ \text { DP01Sum Alt } & Y & \text { U500 } \\ \text { DP30Sum SE } & X & \text { WE } \\ \text { DTn0Sum WE } & X Y & \text { DHi } \\ \text { DTn20 CTI } & X^{2} & \text { HPd } \\ \text { PSpr } & X^{3} & \\ \text { TRan } & Y^{2} & \\ \text { TXInv } & Y^{3} & \\ \text { AET } & X^{2} Y & \\ & X^{2} & \end{array}$

Final favourability model

\begin{tabular}{lrrlll} 
Variables & \multicolumn{1}{c}{ B } & \multicolumn{1}{c}{ SE } & Sig & Evaluation metrics & \\
(Intercept) & $-7.03 \mathrm{E}+00$ & $1.63 \mathrm{E}+00$ & $* * *$ & AUC & 0.9790950 \\
Spatial & $7.69 \mathrm{E}-01$ & $5.21 \mathrm{E}-02$ & $* * *$ & Kappa & 0.6890698 \\
DTn20 & $-5.60 \mathrm{E}-02$ & $1.77 \mathrm{E}-02$ & $* *$ & Sensitivity & 0.9391447 \\
CF & $3.47 \mathrm{E}+00$ & $5.57 \mathrm{E}-01$ & $* * *$ & Specificity & 0.9191489 \\
U100 & $-2.85 \mathrm{E}-02$ & $3.39 \mathrm{E}-03$ & $* * *$ & CCR & 0.921439 \\
Alt & $3.86 \mathrm{E}-03$ & $5.44 \mathrm{E}-04$ & $* * *$ & $\mathrm{HL}$ & $17.4712761 *$ \\
BLF & $2.74 \mathrm{E}+00$ & $5.48 \mathrm{E}-01$ & $* * *$ & & \\
AET & $5.97 \mathrm{E}-03$ & $1.33 \mathrm{E}-03$ & $* * *$ & & \\
TxWin & $2.75 \mathrm{E}-01$ & $9.01 \mathrm{E}-02$ & $* *$ & & \\
SE & $-2.64 \mathrm{E}-02$ & $6.88 \mathrm{E}-03$ & $* * *$ & & \\
DP30Sum & $6.32 \mathrm{E}-01$ & $1.95 \mathrm{E}-01$ & $* *$ & & \\
Sil & $5.14 \mathrm{E}-01$ & $2.11 \mathrm{E}-01 *$ & & \\
NIAL & -1.1542659 & $0.5112433 *$ & &
\end{tabular}




\section{Elaphe scalaris}

Pairwise correlations, spatial descriptor and FDR

Human, lithology and land use variables were not correlated, thus all of them were selected in pairwise correlations.

\section{Variables selected}

$\begin{array}{llll}\text { Climate } & \text { Topography } & \text { Spatial } & \text { Variables excluded by the FDR } \\ \text { DP30Spr } & \text { Alt } & Y & \text { WE } \\ \text { DP30Sum } & \text { SE } & X & \text { SE } \\ \text { DTn0Spr } & \text { WE } & X Y & \text { Calc } \\ \text { DTn0Sum } & \text { CTI } & X^{2} & \text { NIAL } \\ \text { DTn20 } & X^{3} & \text { Clay } \\ \text { DTX25Spr } & Y^{2} & \\ \text { DTX25Sum } & Y^{3} & \\ \text { TRan } & X^{2} Y & \\ \text { ROff } & X^{2} & \\ \text { AET } & & \end{array}$

Final favourability model

\begin{tabular}{lrrlll} 
Variables & \multicolumn{1}{c}{ B } & SE & Sig & Evaluation metrics & \\
(Intercept) & $1.62 \mathrm{E}+00$ & $8.56 \mathrm{E}-01$ & $\mathrm{~ns}$ & AUC & 0.7409146 \\
Spatial & $6.78 \mathrm{E}-01$ & $6.43 \mathrm{E}-02$ & $* * *$ & Kappa & 0.3028314 \\
DTn0Spr & $-4.97 \mathrm{E}-02$ & $5.73 \mathrm{E}-03$ & $* * *$ & Sensitivity & 0.7317588 \\
DTn20 & $-1.16 \mathrm{E}-02$ & $3.16 \mathrm{E}-03$ & $* * *$ & Specificity & 0.5784814 \\
CTI & $-2.74 \mathrm{E}-01$ & $4.37 \mathrm{E}-02$ & $* * *$ & CCR & 0.6469480 \\
HPd & $5.90 \mathrm{E}-04$ & $1.75 \mathrm{E}-04$ & $* * *$ & $\mathrm{HL}$ & $73.9301215 * * *$ \\
DTx25Sum & $2.71 \mathrm{E}-02$ & $5.91 \mathrm{E}-03$ & $* * *$ & & \\
DTx25Spr & $-5.48 \mathrm{E}-02$ & $7.23 \mathrm{E}-03$ & $* * *$ & & \\
U100 & $-7.62 \mathrm{E}-03$ & $1.26 \mathrm{E}-03$ & $* * *$ & & \\
TRan & $1.47 \mathrm{E}-01$ & $2.62 \mathrm{E}-02$ & $* * *$ & & \\
AET & $9.25 \mathrm{E}-04$ & $2.74 \mathrm{E}-04$ & $* * *$ & & \\
Grav & $7.58 \mathrm{E}-01$ & $1.88 \mathrm{E}-01$ & $* * *$ & & \\
Sil & 0.3484844 & 0.0993564 & $* * *$ & & \\
U500 & -0.0019736 & $0.0006779 * *$ & & \\
Past & -3.0309997 & $1.3219593 *$ & & \\
DTn0Sum & 0.4530161 & $0.2208598 *$ & & \\
CF & 0.4398373 & 0.2779301 & $\mathrm{~ns}$ & &
\end{tabular}




\section{Phalacrocorax aristotelis}

Pairwise correlations, spatial descriptor and FDR

Human, lithology and land use variables were not correlated, thus all of them were selected in pairwise correlations.

\section{Variables selected}

$\begin{array}{llll}\text { Climate } & \text { Topography } & \text { Spatial } & \text { Variables excluded by the FDR } \\ \text { DP30Sum } & \text { Alt } & Y & \text { Calc } \\ \text { DTn0Win } & \text { DAlt } & X & \text { WE } \\ \text { DTn0Sum } & \text { SE } & X Y & \text { Shrub } \\ \text { DTn20Aut } & \text { WE } & X^{2} & \\ \text { DTx25Spr } & \text { CTI } & X^{3} & \\ \text { DTx25Sum } & Y^{2} & \\ \text { PAut } & Y^{3} & \\ \text { TRan } & X^{2} Y & \\ \text { AET } & X^{2} & \end{array}$

Final favourability model

$\begin{array}{lrrlll}\text { Variables } & \text { B } & \text { SE } & \text { Sig } & \text { Evaluation metrics } \\ \text { (Intercept) } & 2.99 \mathrm{E}+01 & 5.54 \mathrm{E}+00 & * * * & \text { AUC } & 0.9949604 \\ \text { Spatial } & 5.01 \mathrm{E}-01 & 1.15 \mathrm{E}-01 & * * * & \text { Kappa } & 0.4715157 \\ \text { DTn0Sum } & -2.57 \mathrm{E}+01 & 1.55 \mathrm{E}+01 & \mathrm{~ns} & \text { Sensitivity } & 0.9893617 \\ \text { CTI } & -1.14 \mathrm{E}+00 & 2.95 \mathrm{E}-01 & * * * & \text { Specificity } & 0.9624089 \\ \text { Alt } & -9.30 \mathrm{E}-03 & 2.78 \mathrm{E}-03 & * * * & \text { CCR } & 0.9628862 \\ \text { SE } & -2.11 \mathrm{E}-02 & 1.41 \mathrm{E}-02 & \mathrm{~ns} & \mathrm{HL} & 13.5115766 \mathrm{~ns} \\ \text { U100 } & 5.73 \mathrm{E}-02 & 1.27 \mathrm{E}-02 & * * * & & \\ \text { MF } & -5.01 \mathrm{E}+00 & 1.40 \mathrm{E}+00 & * * * & & \\ \text { TRan } & -6.81 \mathrm{E}-01 & 1.62 \mathrm{E}-01 & * * * & & \\ \text { BLF } & -5.14 \mathrm{E}+00 & 1.43 \mathrm{E}+00 & * * * & & \\ \text { DHi } & -4.19 \mathrm{E}-02 & 1.67 \mathrm{E}-02 & * & & \end{array}$




\section{Ciconia nigra}

Pairwise correlations, spatial descriptor and FDR

Human, lithology and land use variables were not correlated, thus all of them were selected in pairwise correlations.

Variables selected

\begin{tabular}{|c|c|c|c|}
\hline Climate & Topography & Spatial & Variables excluded by the FDR \\
\hline DP10Win & Alt & Y & SE \\
\hline DP30Spr & DAlt & $x$ & CTI \\
\hline DP30Sum & SE & $X Y$ & \\
\hline DTnOSpr & WE & $x^{2}$ & \\
\hline DTnOSum & CTI & $x^{3}$ & \\
\hline DTn20 & & $X^{2} Y$ & \\
\hline DTx25Spr & & $X Y^{2}$ & \\
\hline TRan & & & \\
\hline SIDWin & & & \\
\hline SIDSum & & & \\
\hline AET & & & \\
\hline
\end{tabular}

Final favourability model

\begin{tabular}{lrrlll} 
Variables & \multicolumn{1}{c}{ B } & SE & Sig & Evaluation metrics & \\
(Intercept) & $-2.25 \mathrm{E}+00$ & $1.73 \mathrm{E}+00$ & $\mathrm{~ns}$ & AUC & 0.9619832 \\
Spatial & $6.52 \mathrm{E}-01$ & $7.58 \mathrm{E}-02$ & $* * *$ & Kappa & 0.5469590 \\
AET & $3.92 \mathrm{E}-03$ & $6.99 \mathrm{E}-04$ & $* * *$ & Sensitivity & 0.9539749 \\
HPd & $-1.65 \mathrm{E}-02$ & $7.89 \mathrm{E}-03$ & $*$ & Specificity & 0.8801242 \\
NIAL & $-2.50 \mathrm{E}+00$ & $5.66 \mathrm{E}-01$ & $* * *$ & CCR & 0.8867747 \\
DTn0Sum & $-1.71 \mathrm{E}+00$ & $1.06 \mathrm{E}+00$ & $\mathrm{~ns}$ & $\mathrm{HL}$ & $7.8993221 \mathrm{~ns}$ \\
DHi & $1.71 \mathrm{E}-02$ & $3.29 \mathrm{E}-03$ & $* * *$ & & \\
TRan & $3.21 \mathrm{E}-01$ & $6.73 \mathrm{E}-02$ & $* * *$ & & \\
Sil & $7.39 \mathrm{E}-01$ & $2.72 \mathrm{E}-01$ & $* *$ & & \\
DP30Sum & $-2.33 \mathrm{E}+00$ & $6.52 \mathrm{E}-01$ & $* * *$ & & \\
CF & $2.86 \mathrm{E}+00$ & $7.61 \mathrm{E}-01$ & $* * *$ & & \\
Past & $-3.98 \mathrm{E}+00$ & $2.82 \mathrm{E}+00$ & $\mathrm{~ns}$ & & \\
SIDWin & -3.3990985 & 0.6744347 & $* * *$ & & \\
U500 & -0.0063829 & 0.0017097 & $* * *$ & & \\
DTn0Spr & -0.0340839 & 0.0221235 & $\mathrm{~ns}$ & & \\
BLF & 1.6387195 & 0.6574473 & $*$ & & \\
Alt & -0.0009056 & 0.0005386 & $\mathrm{~ns}$ & & \\
Grav & -0.9810976 & 0.6603876 & $\mathrm{~ns}$ & &
\end{tabular}




\section{Netta rufina}

Pairwise correlations, spatial descriptor and FDR

Human, lithology and land use variables were not correlated, thus all of them were selected in pairwise correlations.

Variables selected

$\begin{array}{llll}\text { Climate } & \text { Topography } & \text { Spatial } & \text { Variables excluded by the FDR } \\ \text { DP30Sum } & \text { Alt } & X Y & \text { Clay } \\ \text { DTnOSum } & \text { DAlt } & X^{2} & \text { NIAL } \\ \text { DTn20 } & \text { SE } & X^{3} & \text { HPd } \\ \text { TRan } & \text { WE } & Y^{2} & \text { TRan } \\ \text { SIDSpr } & \text { CTI } & Y^{3} & \text { Gyps } \\ \text { AET } & & X^{2} Y & \text { SE } \\ & & X Y^{2} & \text { WE }\end{array}$

Final favourability model

\begin{tabular}{lrrlll}
\multicolumn{1}{c}{ Variables } & B & SE & Sig & Evaluation metrics \\
(Intercept) & $-1.73 \mathrm{E}+01$ & $2.54 \mathrm{E}+00$ & $* * *$ & AUC & 0.9130220 \\
CTI & $5.94 \mathrm{E}-01$ & $1.03 \mathrm{E}-01$ & $* * *$ & Kappa & 0.1535811 \\
Spatial & $4.89 \mathrm{E}-01$ & $9.27 \mathrm{E}-02$ & $* * *$ & Sensitivity & 0.9113924 \\
SIDSpr & $1.24 \mathrm{E}+00$ & $5.06 \mathrm{E}-01$ & $*$ & Specificity & 0.7768932 \\
MF & $-1.99 \mathrm{E}+01$ & $1.50 \mathrm{E}+01$ & $\mathrm{~ns}$ & CCR & 0.7808968 \\
Grav & $7.86 \mathrm{E}-01$ & $3.83 \mathrm{E}-01$ & $*$ & $\mathrm{HL}$ & $11.3447743 \mathrm{~ns}$ \\
CF & $-3.69 \mathrm{E}+00$ & $1.53 \mathrm{E}+00$ & $*$ & & \\
BLF & $-4.74 \mathrm{E}+00$ & $2.71 \mathrm{E}+00$ & $\mathrm{~ns}$ & & \\
Sil & $-1.18 \mathrm{E}+00$ & $4.70 \mathrm{E}-01$ & $*$ & & \\
DHi & $1.33 \mathrm{E}-02$ & $5.48 \mathrm{E}-03$ & $*$ & & \\
DTn20 & $9.35 \mathrm{E}-03$ & $5.89 \mathrm{E}-03$ & $\mathrm{~ns}$ & &
\end{tabular}




\section{Gypaetus barbatus}

Pairwise correlations, spatial descriptor and FDR

Human, lithology and land use variables were not correlated, thus all of them were selected in pairwise correlations.

\section{Variables selected}

$\begin{array}{llll}\text { Climate } & \text { Topography } & \text { Spatial } & \text { Variables excluded by the FDR } \\ \text { DP10Spr } & \text { Alt } & X & \text { Sil } \\ \text { DP10Sum } & \text { DAlt } & X^{3} & \text { WE } \\ \text { DTnOWin } & \text { SE } & Y^{2} & \text { U500 } \\ \text { DTn0Sum } & \text { WE } & Y^{3} & \text { Gyps } \\ \text { DTn20Aut } & \text { CTI } & X^{2} & \text { TRan } \\ \text { TRan } & & \\ \text { AET } & & & \end{array}$

Final favourability model

\begin{tabular}{|c|c|c|c|c|c|}
\hline Variables & B & SE & Sig & Evaluation metrics & \\
\hline (Intercept) & $4.09 E+01$ & $1.25 \mathrm{E}+01$ & $* *$ & AUC & 0.9952292 \\
\hline Spatial & $1.20 \mathrm{E}+00$ & 1.87E-01 & $* * *$ & Карра & 0.5098472 \\
\hline DAlt & $3.65 \mathrm{E}-03$ & 7.99E-04 & $* * *$ & Sensitivity & 1 \\
\hline U100 & $-2.45 \mathrm{E}-02$ & $1.02 \mathrm{E}-02$ & * & Specificity & 0.9687680 \\
\hline Shrub & $5.17 \mathrm{E}+00$ & $1.61 \mathrm{E}+00$ & $* *$ & CCR & 0.9692916 \\
\hline CTI & $-2.62 E+00$ & 7.10E-01 & $* * *$ & $\mathrm{HL}$ & $9.6854071 \mathrm{~ns}$ \\
\hline DP10Sum & $-4.18 \mathrm{E}-01$ & $1.96 \mathrm{E}-01$ & $*$ & & \\
\hline SE & $3.98 \mathrm{E}-02$ & $1.95 \mathrm{E}-02$ & $*$ & & \\
\hline MF & $4.22 \mathrm{E}+00$ & $1.84 \mathrm{E}+00$ & $*$ & & \\
\hline CF & $-2.00 E+00$ & $1.27 \mathrm{E}+00$ & ns & & \\
\hline DTnOWin & $-3.14 \mathrm{E}-02$ & 1.34E-02 & $*$ & & \\
\hline
\end{tabular}




\section{Neophron percnopterus}

Pairwise correlations, spatial descriptor and FDR

Human, lithology and land use variables were not correlated, thus all of them were selected in pairwise correlations.

\section{Variables selected}

$\begin{array}{llll}\text { Climate } & \text { Topography } & \text { Spatial } & \text { Variables excluded by the FDR } \\ \text { DP10Sum } & \text { Alt } & X Y & \text { WE } \\ \text { DP30Spr } & \text { DAlt } & X^{2} & \text { TRan } \\ \text { DTn0Sum } & \text { SE } & X^{3} & \text { U500 } \\ \text { DTn20 } & \text { WE } & Y^{2} & \text { Sil } \\ \text { TRan } \quad \text { CTI } & Y^{3} & \\ \text { TXWin } & & X^{2} Y & \\ \text { SISWin } & X^{2} & \\ \text { ROff } & & & \\ \text { AET } & & \end{array}$

Final favourability model

\begin{tabular}{lrrlll} 
Variables & \multicolumn{1}{c}{ B } & \multicolumn{1}{c}{ SE } & Sig & Evaluation metrics & \\
(Intercept) & $6.94 \mathrm{E}+00$ & $1.96 \mathrm{E}+00$ & $* * *$ & AUC & 0.8543394 \\
Spatial & $1.16 \mathrm{E}+00$ & $6.99 \mathrm{E}-02$ & $* * *$ & Kappa & 0.3870012 \\
Shrub & $2.45 \mathrm{E}+00$ & $2.76 \mathrm{E}-01$ & $* * *$ & Sensitivity & 0.7877155 \\
DHi & $1.54 \mathrm{E}-02$ & $2.11 \mathrm{E}-03$ & $* * *$ & Specificity & 0.7481735 \\
BLF & $1.78 \mathrm{E}+00$ & $3.77 \mathrm{E}-01$ & $* * *$ & CCR & 0.7550867 \\
DTn20 & $1.25 \mathrm{E}-02$ & $5.42 \mathrm{E}-03$ & $*$ & $\mathrm{HL}$ & $72.8182265 * * *$ \\
Calc & $9.70 \mathrm{E}-01$ & $1.44 \mathrm{E}-01$ & $* * *$ & & \\
U100 & $1.08 \mathrm{E}-02$ & $1.70 \mathrm{E}-03$ & $* * *$ & & \\
MF & $2.36 \mathrm{E}+00$ & $5.70 \mathrm{E}-01$ & $* * *$ & & \\
SE & $-1.72 \mathrm{E}-02$ & $3.63 \mathrm{E}-03$ & $* * *$ & & \\
DAlt & $1.30 \mathrm{E}-03$ & $2.43 \mathrm{E}-04$ & $* * *$ & & \\
DP10Sum & $-2.12 \mathrm{E}-01$ & $4.63 \mathrm{E}-02$ & $* * *$ & & \\
Alt & -0.0021521 & 0.0002819 & $* * *$ & & \\
TxWin & -0.2564922 & 0.0442536 & $* * *$ & & \\
CTI & -0.2198655 & 0.095337 & $*$ & & \\
DP30Spr & -0.2524642 & 0.0885915 & $* *$ & & \\
NIAL & -0.679976 & 0.271057 & $*$ & & \\
Clay & 0.429391 & $0.1893503 *$ & & \\
AET & 0.0009067 & $0.0004482 *$ & & \\
Gyps & 0.6010296 & 0.3554959 & $\mathrm{~ns}$ & &
\end{tabular}




\section{Aegypus monachus}

Pairwise correlations, spatial descriptor and FDR

Human, lithology and land use variables were not correlated, thus all of them were selected in pairwise correlations.

Variables selected

$\begin{array}{llll}\text { Climate } & \text { Topography } & \text { Spatial } & \text { Variables excluded by the FDR } \\ \text { DP30Spr } & \text { Alt } & X & \text { DAlt } \\ \text { DP30Sum } & \text { DAlt } & X Y & \text { CF } \\ \text { DTn0Sum SE } & X^{2} & \text { WE } \\ \text { DTn20 WE } & X^{3} & \text { DP30Spr } \\ \text { DTX25Spr CTI } & Y^{2} & \text { Alt } \\ \text { TRan } & Y^{3} & \text { SE } \\ \text { TnJul } & X^{2} & \\ \text { SIDWin } & & \\ \text { SIDSum } & & \\ \text { AET } & & \end{array}$

Final favourability model

\begin{tabular}{|c|c|c|c|c|c|}
\hline Variables & ß & SE & Sig & \multicolumn{2}{|c|}{ Evaluation metrics } \\
\hline (Intercept) & $6.12 E+00$ & $4.42 \mathrm{E}+00$ & ns & AUC & 0.9668476 \\
\hline Spatial & $1.08 \mathrm{E}+00$ & $1.64 \mathrm{E}-01$ & $* * *$ & Kappa & 0.2833218 \\
\hline Shrub & $2.55 \mathrm{E}+00$ & $6.09 \mathrm{E}-01$ & $* * *$ & Sensitivity & 0.9615385 \\
\hline BLF & $3.47 E+00$ & 9.23E-01 & $* * *$ & Specificity & 0.8765528 \\
\hline $\mathrm{DHi}$ & $2.12 \mathrm{E}-02$ & 4.62E-03 & $* * *$ & CCR & 0.8790505 \\
\hline NIAL & $-2.03 E+00$ & $1.22 \mathrm{E}+00$ & ns & $\mathrm{HL}$ & $8.0547909 \mathrm{~ns}$ \\
\hline DTx25Spr & $6.49 E-02$ & $2.00 \mathrm{E}-02$ & $* *$ & & \\
\hline SIDSum & $-2.95 E+00$ & 7.08E-01 & $* * *$ & & \\
\hline U100 & $-1.18 \mathrm{E}-02$ & $3.82 \mathrm{E}-03$ & $* *$ & & \\
\hline Past & $-1.25 \mathrm{E}+01$ & $6.61 E+00$ & ns & & \\
\hline $\mathrm{HPd}$ & $-2.20 \mathrm{E}-02$ & $1.88 \mathrm{E}-02$ & ns & & \\
\hline AET & $4.00 \mathrm{E}-03$ & $1.20 \mathrm{E}-03$ & $* * *$ & & \\
\hline TRan & 0.373362 & 0.11373 & $* *$ & & \\
\hline Calc & -2.011601 & 0.92926 & * & & \\
\hline Grav & -2.374854 & 1.579367 & ns & & \\
\hline DTn20 & -0.017868 & 0.012335 & ns & & \\
\hline
\end{tabular}




\section{Aquila adalberti}

Pairwise correlations, spatial descriptor and FDR

Human, lithology and land use variables were not correlated, thus all of them were selected in pairwise correlations.

Variables selected

$\begin{array}{llll}\text { Climate } & \text { Topography } & \text { Spatial } & \text { Variables excluded by the FDR } \\ \text { DP30Spr } & \text { Alt } & Y & \text { Slop } \\ \text { DP30Sum } & \text { Slop } & X & \text { HPd } \\ \text { DTn0Sum } & \text { SE } & \text { XY } & \text { Alt } \\ \text { DTn20 WE } & X^{2} & \text { SE } \\ \text { DTx25Spr } & X^{3} & \text { CF } \\ \text { TRan } & Y^{2} & \text { U500 } \\ \text { TnJul } & Y^{3} & \text { WE } \\ \text { SIDWin } & X^{2} Y & \\ \text { SIDSum } & X^{2} & \\ \text { AET } & & \end{array}$

Final favourability model

\begin{tabular}{lrrlll} 
Variables & \multicolumn{1}{c}{ B } & \multicolumn{1}{c}{ SE } & Sig & Evaluation metrics & \\
(Intercept) & $1.48 \mathrm{E}+01$ & $5.08 \mathrm{E}+00$ & $* *$ & AUC & 0.9509119 \\
Spatial & $1.06 \mathrm{E}+00$ & $1.31 \mathrm{E}-01$ & $* * *$ & Kappa & 0.235036 \\
NIAL & $-3.66 \mathrm{E}+00$ & $8.89 \mathrm{E}-01$ & $* * *$ & Sensitivity & 0.9556962 \\
Shrub & $1.49 \mathrm{E}+00$ & $5.45 \mathrm{E}-01$ & $* *$ & Specificity & 0.8460194 \\
DP30Spr & $-1.27 \mathrm{E}+00$ & $2.83 \mathrm{E}-01$ & $* * *$ & CCR & 0.8492841 \\
BLF & $3.37 \mathrm{E}+00$ & $9.13 \mathrm{E}-01$ & $* * *$ & HL & $1.9422848 \mathrm{~ns}$ \\
DHi & $-7.26 \mathrm{E}-03$ & $4.36 \mathrm{E}-03$ & $\mathrm{~ns}$ & & \\
AET & $4.47 \mathrm{E}-03$ & $1.13 \mathrm{E}-03$ & $* * *$ & & \\
Calc & $-1.97 \mathrm{E}+00$ & $6.75 \mathrm{E}-01$ & $* *$ & & \\
Clay & $-1.40 \mathrm{E}+00$ & $5.11 \mathrm{E}-01$ & $* *$ & & \\
TRan & $2.50 \mathrm{E}-01$ & $8.61 \mathrm{E}-02$ & $* *$ & & \\
SIDSum & $-4.73 \mathrm{E}+00$ & $1.03 \mathrm{E}+00$ & $* * *$ & & \\
SIDWin & 1.75943 & 1.003337 & $\mathrm{~ns}$ & & \\
Past & -18.897838 & 11.427461 & $\mathrm{~ns}$ & & \\
Grav & 1.205273 & 0.555369 & $*$ & & \\
DTx25Spr & 0.041297 & 0.020827 & $*$ & & \\
DTn20 & -0.030661 & 0.014351 & $*$ & & \\
TnJul & 0.154892 & 0.098609 & $\mathrm{~ns}$ & &
\end{tabular}




\section{Aquila chrysaetos}

Pairwise correlations, spatial descriptor and FDR

Human, lithology and land use variables were not correlated, thus all of them were selected in pairwise correlations.

\section{Variables selected}

$\begin{array}{llll}\text { Climate } & \text { Topography } & \text { Spatial } & \text { Variables excluded by the FDR } \\ \text { DP01Win } & \text { Alt } & Y & \text { WE } \\ \text { DP30Spr } & \text { DAlt } & X & \text { Gyps } \\ \text { DTn0Sum } & \text { SE } & X Y & \text { U500 } \\ \text { DTn20Aut } & \text { WE } & X^{2} & \text { MF } \\ \text { TSum } & \text { CTI } & X^{3} & \text { SE } \\ \text { TRan } & Y^{2} & \\ \text { TnWin } & Y^{3} & \\ \text { AET } & X^{2} Y & \\ & X^{2} & \end{array}$

Final favourability model

\begin{tabular}{lrrlll} 
Variables & \multicolumn{1}{c}{ B } & \multicolumn{1}{c}{ SE } & Sig & Evaluation metrics & \\
(Intercept) & $5.57 \mathrm{E}+00$ & $1.72 \mathrm{E}+00$ & $* *$ & AUC & 0.8345716 \\
CTI & $-5.37 \mathrm{E}-01$ & $9.21 \mathrm{E}-02$ & $* * *$ & Kappa & 0.4719186 \\
TRan & $3.63 \mathrm{E}-01$ & $7.22 \mathrm{E}-02$ & $* * *$ & Sensitivity & 0.7692308 \\
Shrub & $2.38 \mathrm{E}+00$ & $2.22 \mathrm{E}-01$ & $* * *$ & Specificity & 0.7506717 \\
Alt & $6.41 \mathrm{E}-04$ & $2.26 \mathrm{E}-04$ & $* *$ & CCR & 0.7562170 \\
DAlt & $1.55 \mathrm{E}-03$ & $2.10 \mathrm{E}-04$ & $* * *$ & $\mathrm{HL}$ & $28.4659447 * * *$ \\
Spatial & $6.38 \mathrm{E}-01$ & $9.12 \mathrm{E}-02$ & $* * *$ & & \\
DP30Spr & $-4.33 \mathrm{E}-01$ & $7.61 \mathrm{E}-02$ & $* * *$ & & \\
DHi & $6.83 \mathrm{E}-03$ & $1.74 \mathrm{E}-03$ & $* * *$ & & \\
BLF & $1.99 \mathrm{E}+00$ & $3.38 \mathrm{E}-01$ & $* * *$ & & \\
Past & $-4.39 \mathrm{E}+00$ & $1.22 \mathrm{E}+00$ & $* * *$ & & \\
CF & $1.27 \mathrm{E}+00$ & $3.03 \mathrm{E}-01$ & $* * *$ & & \\
Clay & -0.5084866 & 0.1495641 & $* * *$ & & \\
HPd & -0.0011865 & 0.000636 & $\mathrm{~ns}$ & & \\
AET & -0.0009261 & 0.0003611 & $*$ & & \\
Grav & -0.4254798 & 0.2645641 & $\mathrm{~ns}$ & & \\
TnWin & 0.2504006 & 0.0678657 & $* * *$ & & \\
TSum & -0.1988456 & $0.0703953 * *$ & & \\
DTn20Aut & -0.0401276 & 0.0191527 & $*$ & &
\end{tabular}




\section{Lagopus mutus}

Pairwise correlations, spatial descriptor and FDR

Human, lithology and land use variables were not correlated, thus all of them were selected in pairwise correlations.

Variables selected

$\begin{array}{llll}\text { Climate } & \text { Topography } & \text { Spatial } & \text { Variables excluded by the FDR } \\ \text { DP10Spr } & \text { Alt } & Y & \text { Past } \\ \text { DTn0 } & \text { SE } & X Y & \text { Calc } \\ \text { DTn0Sum } & \text { WE } & X^{2} & \text { WE } \\ \text { DTn20 } & \text { CTI } & X^{3} & \text { MF } \\ \text { TSum } & & Y^{3} & \\ \text { TRan } & & X^{2} & \\ \text { AET } & & & \end{array}$

Final favourability model

$\begin{array}{lrrlll}\text { Variables } & \text { B } & \text { SE } & \text { Sig } & \text { Evaluation metrics } \\ \text { (Intercept) } & -1.09 \mathrm{E}+01 & 3.23 \mathrm{E}+00 & * * * & \text { AUC } & 0.9994035 \\ \text { Spatial } & 4.61 \mathrm{E}-01 & 1.81 \mathrm{E}-01 & * & \text { Kappa } & 0.7386766 \\ \text { DTn0 } & 5.79 \mathrm{E}-02 & 1.53 \mathrm{E}-02 & * * * & \text { Sensitivity } & 1 \\ \text { DP10Spr } & 1.85 \mathrm{E}-01 & 1.22 \mathrm{E}-01 & \mathrm{~ns} & \text { Specificity } & 0.9918033 \\ \text { Clay } & -1.15 \mathrm{E}+01 & 6.38 \mathrm{E}+00 & \mathrm{~ns} & \text { CCR } & 0.9918990 \\ \text { HPd } & -3.77 \mathrm{E}-01 & 2.80 \mathrm{E}-01 & \mathrm{~ns} & \mathrm{HL} & 10.9099107 \text { ns }\end{array}$




\section{Tetrao urogallus}

Pairwise correlations, spatial descriptor and FDR

Human, lithology and land use variables were not correlated, thus all of them were selected in pairwise correlations.

\section{Variables selected}

$\begin{array}{llll}\text { Climate } & \text { Topography } & \text { Spatial } & \text { Variables excluded by the FDR } \\ \text { DP10Spr } & \text { Alt } & Y & \text { SE } \\ \text { DP10Sum SE } & X & \text { Calc } \\ \text { DTn0Sum } & \text { WE } & X Y & \text { WE } \\ \text { DTn20 CTI } & X^{2} & \\ \text { Tann } & X^{3} & \\ \text { TRan } & Y^{2} & \\ \text { AET } & X^{2} Y & \\ & & X Y^{2} & \end{array}$

Final favourability model

\begin{tabular}{lrrlll} 
Variables & \multicolumn{1}{c}{ B } & SE & Sig & Evaluation metrics & \\
(Intercept) & $2.57 \mathrm{E}+01$ & $9.73 \mathrm{E}+00$ & $* *$ & AUC & 0.9952019 \\
CTI & $-2.21 \mathrm{E}+00$ & $5.55 \mathrm{E}-01$ & $* * *$ & Kappa & 0.5690619 \\
Spatial & $7.09 \mathrm{E}-01$ & $1.25 \mathrm{E}-01$ & $* * *$ & Sensitivity & 0.9793103 \\
CF & $1.10 \mathrm{E}+01$ & $1.68 \mathrm{E}+00$ & $* * *$ & Specificity & 0.9616502 \\
U500 & $2.57 \mathrm{E}-02$ & $4.15 \mathrm{E}-03$ & $* * *$ & CCR & 0.9621326 \\
TRan & $3.29 \mathrm{E}-01$ & $1.20 \mathrm{E}-01$ & $* *$ & HL & $8.3280171 \mathrm{~ns}$ \\
AET & $-1.29 \mathrm{E}-02$ & $4.41 \mathrm{E}-03$ & $* *$ & & \\
BLF & $8.13 \mathrm{E}+00$ & $1.45 \mathrm{E}+00$ & $* * *$ & & \\
Sil & $-2.39 \mathrm{E}+00$ & $5.62 \mathrm{E}-01$ & $* * *$ & & \\
MF & $7.24 \mathrm{E}+00$ & $1.61 \mathrm{E}+00$ & $* * *$ & & \\
Shrub & $6.44 \mathrm{E}+00$ & $1.45 \mathrm{E}+00$ & $* * *$ & & \\
Alt & $4.13 \mathrm{E}-03$ & $8.59 \mathrm{E}-04$ & $* * *$ & & \\
DTn0Sum & -0.9831937 & 0.3410507 & $* *$ & & \\
Past & 6.9874094 & $3.1664896 *$ & & \\
Grav & -6.5479217 & 4.8726068 & $\mathrm{~ns}$ & &
\end{tabular}




\section{Perdix perdix}

Pairwise correlations, spatial descriptor and FDR

Human, lithology and land use variables were not correlated, thus all of them were selected in pairwise correlations.

\section{Variables selected}

$\begin{array}{llll}\text { Climate } & \text { Topography } & \text { Spatial } & \text { Variables excluded by the FDR } \\ \text { DP10Spr } & \text { Alt } & Y & \text { SE } \\ \text { DP30Sum SE } & X Y & \text { WE } \\ \text { DTnOSum } & \text { WE } & X^{2} & \\ \text { DTn20 CTI } & X^{3} & \\ \text { TSum } & Y^{2} & \\ \text { TRan } & Y^{3} & \\ \text { TXWin } & X^{2} Y & \\ \text { SIDSum } & X Y^{2} & \\ \text { AET } & \end{array}$

Final favourability model

\begin{tabular}{lrclll}
\multicolumn{1}{c}{ Variables } & B & SE & Sig & Evaluation metrics & \\
(Intercept) & $2.92 \mathrm{E}+01$ & $6.72 \mathrm{E}+00$ & $* * *$ & AUC & 0.9891759 \\
CTI & $-2.01 \mathrm{E}+00$ & $3.17 \mathrm{E}-01$ & $* * *$ & Kappa & 0.5682411 \\
Spatial & $4.77 \mathrm{E}-01$ & $8.68 \mathrm{E}-02$ & $* * *$ & Sensitivity & 0.9686099 \\
AET & $-1.13 \mathrm{E}-02$ & $2.56 \mathrm{E}-03$ & $* * *$ & Specificity & 0.9431662 \\
DTn20 & $-1.67 \mathrm{E}-01$ & $7.06 \mathrm{E}-02$ & $*$ & CCR & 0.9442351 \\
BLF & $5.62 \mathrm{E}+00$ & $9.43 \mathrm{E}-01$ & $* * *$ & $\mathrm{HL}$ & $10.7791335 \mathrm{~ns}$ \\
Shrub & $5.16 \mathrm{E}+00$ & $9.32 \mathrm{E}-01$ & $* * *$ & & \\
Alt & $4.36 \mathrm{E}-03$ & $1.01 \mathrm{E}-03$ & $* * *$ & & \\
Past & $5.13 \mathrm{E}+00$ & $1.68 \mathrm{E}+00$ & $* *$ & & \\
MF & $3.16 \mathrm{E}+00$ & $1.26 \mathrm{E}+00$ & $*$ & & \\
CF & $2.68 \mathrm{E}+00$ & $9.51 \mathrm{E}-01$ & $* *$ & & \\
U100 & $-1.42 \mathrm{E}-02$ & $5.70 \mathrm{E}-03 *$ & & \\
TxWin & 0.366997 & 0.162182 & $*$ & & \\
DTn0Sum & 0.585695 & $0.257855 *$ & & \\
DHi & 0.016267 & $0.007717 *$ & & \\
DP30Sum & -0.618032 & $0.255162 *$ & &
\end{tabular}




\section{Otis tarda}

Pairwise correlations, spatial descriptor and FDR

Human, lithology and land use variables were not correlated, thus all of them were selected in pairwise correlations.

\section{Variables selected}

$\begin{array}{llll}\text { Climate } & \text { Topography } & \text { Spatial } & \text { Variables excluded by the FDR } \\ \text { DP30Spr } & \text { Alt } & Y & \text { U100 } \\ \text { DP30Sum } & \text { Slop } & X & \text { Calc } \\ \text { DTnOSum } & \text { SE } & X Y & \text { DHi } \\ \text { DTn20Aut } & \text { WE } & X^{2} & \\ \text { TRan } & X^{3} & \\ \text { TXSpr } & X^{2} Y & \\ \text { SIDSum } & X^{2} & \\ \text { SISWin } & & \\ \text { ROff } & & \\ \text { AET } & & \end{array}$

Final favourability model

$\begin{array}{lrrlll}\text { Variables } & \text { B } & \text { SE } & \text { Sig } & \text { Evaluation metrics } & \\ \text { (Intercept) } & -7.15 \mathrm{E}+00 & 1.74 \mathrm{E}+00 & * * * & \text { AUC } & 0.9215608 \\ \text { Slop } & -7.61 \mathrm{E}-01 & 8.38 \mathrm{E}-02 & * * * & \text { Kappa } & 0.3883926 \\ \text { NIAL } & 2.84 \mathrm{E}+00 & 2.19 \mathrm{E}-01 & * * * & \text { Sensitivity } & 0.8863636 \\ \text { SIDSum } & 5.07 \mathrm{E}-01 & 3.01 \mathrm{E}-01 & \mathrm{~ns} & \text { Specificity } & 0.7981172 \\ \text { Spatial } & 4.08 \mathrm{E}-01 & 8.14 \mathrm{E}-02 & * * * & \text { CCR } & 0.8068953 \\ \text { CF } & -5.10 \mathrm{E}+00 & 1.36 \mathrm{E}+00 & * * * & \mathrm{HL} & 5.4435670 \mathrm{~ns} \\ \text { TRan } & 1.97 \mathrm{E}-01 & 5.05 \mathrm{E}-02 & * * * & & \\ \text { HPd } & -1.80 \mathrm{E}-03 & 7.60 \mathrm{E}-04 & * & & \\ \text { Gyps } & -4.07 \mathrm{E}+00 & 2.26 \mathrm{E}+00 & \mathrm{~ns} & & \\ \text { AET } & -1.80 \mathrm{E}-03 & 5.90 \mathrm{E}-04 & * * & & \\ \text { DTn20Aut } & 7.27 \mathrm{E}-02 & 2.88 \mathrm{E}-02 & * & & \\ \text { DTn0Sum } & -2.62 \mathrm{E}+00 & 1.27 \mathrm{E}+00 & * & & \\ \text { U500 } & 0.0024771 & 0.0010579 & & & \\ \text { WE } & 0.0079399 & 0.0054528 & \mathrm{~ns} & & \end{array}$




\section{Picus viridis}

Pairwise correlations, spatial descriptor and FDR

Human, lithology and land use variables were not correlated, thus all of them were selected in pairwise correlations.

\section{Variables selected}

$\begin{array}{llll}\text { Climate } & \text { Topography } & \text { Spatial } & \text { Variables excluded by the FDR } \\ \text { DP1Sum } & \text { Alt } & X & \text { HPd } \\ \text { DP30Sum } & \text { DAIt } & X Y & \text { NIAL } \\ \text { DTnOSum } & \text { SE } & X^{2} & \text { Clay } \\ \text { DTn20 } & \text { WE } & Y^{2} & \\ \text { PSpr } & \text { CTI } & Y^{3} & \\ \text { TAut } & & X^{2} Y & \\ \text { TRan } & & X^{2} & \\ \text { AET } & & & \end{array}$

Final favourability model

\begin{tabular}{lrrlll} 
Variables & \multicolumn{1}{c}{ B } & \multicolumn{1}{c}{ SE } & Sig & Evaluation metrics & \\
(Intercept) & $-7.51 \mathrm{E}+00$ & $1.87 \mathrm{E}+00$ & $* * *$ & AUC & 0.8369539 \\
Spatial & $8.82 \mathrm{E}-01$ & $7.67 \mathrm{E}-02$ & $* * *$ & Kappa & 0.4148709 \\
DAlt & $1.87 \mathrm{E}-03$ & $2.86 \mathrm{E}-04$ & $* * *$ & Sensitivity & 0.7716463 \\
DTn20 & $-3.20 \mathrm{E}-02$ & $4.47 \mathrm{E}-03$ & $* * *$ & Specificity & 0.7686796 \\
DTn0Sum & $-1.36 \mathrm{E}+00$ & $2.08 \mathrm{E}-01$ & $* * *$ & CCR & 0.7711002 \\
BLF & $3.15 \mathrm{E}+00$ & $5.14 \mathrm{E}-01$ & $* * *$ & HL & $24.7656540 * *$ \\
PSpr & $-2.86 \mathrm{E}-03$ & $9.88 \mathrm{E}-04$ & $* *$ & & \\
MF & $3.67 \mathrm{E}+00$ & $9.30 \mathrm{E}-01$ & $* * *$ & & \\
CF & $1.82 \mathrm{E}+00$ & $4.73 \mathrm{E}-01$ & $* * *$ & & \\
DHi & $-8.08 \mathrm{E}-03$ & $2.19 \mathrm{E}-03$ & $* * *$ & & \\
Shrub & $1.09 \mathrm{E}+00$ & $2.64 \mathrm{E}-01$ & $* * *$ & & \\
Alt & $1.76 \mathrm{E}-03$ & $3.35 \mathrm{E}-04$ & $* * *$ & & \\
SE & 0.0097427 & 0.0031684 & $* *$ & & \\
U500 & -0.0014572 & 0.0008354 & $\mathrm{~ns}$ & & \\
Gyps & 2.0835307 & 0.7763705 & $* *$ & & \\
TAut & 0.1962981 & 0.0604723 & $* *$ & & \\
U100 & -0.0036382 & 0.0015055 & $*$ & & \\
CTI & 0.1801179 & 0.0690682 & $* *$ & & \\
Sil & 0.4071759 & 0.1650496 & $*$ & & \\
Calc & 0.3333775 & 0.1835942 & $\mathrm{~ns}$ & &
\end{tabular}




\section{Pyrrhocorax graculus}

Pairwise correlations, spatial descriptor and FDR

Human, lithology and land use variables were not correlated, thus all of them were selected in pairwise correlations.

\section{Variables selected}

$\begin{array}{llll}\text { Climate } & \text { Topography } & \text { Spatial } & \text { Variables excluded by the FDR } \\ \text { DTnOSum } & \text { Alt } & Y & \text { U500 } \\ \text { DTn20 } & \text { SE } & X Y & \text { SE } \\ \text { PSpr } & \text { WE } & X^{2} & \text { Shrub } \\ \text { TJul } & \text { CTI } & Y^{2} & \text { WE } \\ \text { TRan } & & Y^{3} & \\ \text { TXWin } & X^{2} Y & \\ \text { AET } & & X^{2} & \end{array}$

Final favourability model

\begin{tabular}{lrclll}
\multicolumn{1}{c}{ Variables } & B & SE & Sig & Evaluation metrics \\
(Intercept) & $3.38 \mathrm{E}+01$ & $4.96 \mathrm{E}+00$ & $* * *$ & AUC & 0.9896141 \\
Spatial & $4.63 \mathrm{E}-01$ & $8.37 \mathrm{E}-02$ & $* * *$ & Kappa & 0.5892420 \\
CTI & $-1.88 \mathrm{E}+00$ & $2.70 \mathrm{E}-01$ & $* * *$ & Sensitivity & 0.9879518 \\
Calc & $3.42 \mathrm{E}+00$ & $4.01 \mathrm{E}-01$ & $* * *$ & Specificity & 0.9399091 \\
TJul & $-6.38 \mathrm{E}-01$ & $1.16 \mathrm{E}-01$ & $* * *$ & CCR & 0.9421628 \\
DTn0Sum & $9.52 \mathrm{E}-01$ & $3.32 \mathrm{E}-01$ & $* *$ & $\mathrm{HL}$ & $9.9494821 \mathrm{~ns}$ \\
CF & $-3.10 \mathrm{E}+00$ & $8.84 \mathrm{E}-01$ & $* * *$ & & \\
TRan & $1.77 \mathrm{E}-01$ & $7.33 \mathrm{E}-02$ & $*$ & & \\
AET & $7.33 \mathrm{E}-03$ & $3.54 \mathrm{E}-03$ & $*$ & & \\
PSpr & $-3.06 \mathrm{E}-03$ & $1.79 \mathrm{E}-03$ & $\mathrm{~ns}$ & &
\end{tabular}




\section{Pyrrhocorax pyrrhocorax}

Pairwise correlations, spatial descriptor and FDR

Human, lithology and land use variables were not correlated, thus all of them were selected in pairwise correlations.

\section{Variables selected}

$\begin{array}{llll}\text { Climate } & \text { Topography } & \text { Spatial } & \text { Variables excluded by the FDR } \\ \text { DP01Win } & \text { Alt } & Y & \text { WE } \\ \text { DP10Sum } & \text { DAlt } & X Y & \text { Past } \\ \text { DTnOSum } & \text { SE } & X^{2} & \text { AET } \\ \text { DTn20Aut } & \text { WE } & X^{3} & \text { TRan } \\ \text { PSpr } & \text { CTI } & Y^{2} & \text { DP01Win } \\ \text { TAut } & X^{2} Y & \text { Clay } \\ \text { TRan } & X^{2} & \\ \text { AET } & & & \end{array}$

Final favourability model

\begin{tabular}{lrrlll} 
Variables & \multicolumn{1}{c}{ B } & SE & Sig & Evaluation metrics & \\
(Intercept) & $1.35 \mathrm{E}+01$ & $1.79 \mathrm{E}+00$ & $* * *$ & AUC & 0.8667009 \\
Spatial & $9.11 \mathrm{E}-01$ & $4.72 \mathrm{E}-02$ & $* * *$ & Kappa & 0.5460969 \\
DAlt & $1.61 \mathrm{E}-03$ & $2.08 \mathrm{E}-04$ & $* * *$ & Sensitivity & 0.8204986 \\
Shrub & $1.34 \mathrm{E}+00$ & $2.34 \mathrm{E}-01$ & $* * *$ & Specificity & 0.7627748 \\
HPd & $-9.40 \mathrm{E}-04$ & $4.01 \mathrm{E}-04$ & $*$ & CCR & 0.7824039 \\
SE & $-1.32 \mathrm{E}-02$ & $2.91 \mathrm{E}-03$ & $* * *$ & $\mathrm{HL}$ & $26.6660089 * * *$ \\
PSpr & $-3.90 \mathrm{E}-03$ & $7.92 \mathrm{E}-04$ & $* * *$ & & \\
DTn20Aut & $-1.02 \mathrm{E}-01$ & $2.00 \mathrm{E}-02$ & $* * *$ & & \\
CTI & $-6.27 \mathrm{E}-01$ & $9.20 \mathrm{E}-02$ & $* * *$ & & \\
CF & $-1.57 \mathrm{E}+00$ & $3.06 \mathrm{E}-01$ & $* * *$ & & \\
U100 & $6.33 \mathrm{E}-03$ & $1.49 \mathrm{E}-03$ & $* * *$ & & \\
TAut & $-1.23 \mathrm{E}-01$ & $2.29 \mathrm{E}-02$ & $* * *$ & & \\
DP10Sum & -0.1288606 & 0.0429071 & $* *$ & & \\
DHi & 0.005302 & 0.0020781 & & & \\
U500 & -0.0014488 & $0.0007078 *$ & & \\
Calc & 0.4651237 & 0.1302131 & $* * *$ & & \\
Grav & 0.6346786 & $0.2509335 *$ & &
\end{tabular}




\section{Galemys pyrenaicus}

Pairwise correlations, spatial descriptor and FDR

Human, lithology and land use variables were not correlated, thus all of them were selected in pairwise correlations.

\section{Variables selected}

$\begin{array}{llll}\text { Climate } & \text { Topography } & \text { Spatial } & \text { Variables excluded by the FDR } \\ \text { DP30Sum } & \text { Alt } & X & \text { SE } \\ \text { DTnOSum } & \text { DAlt } & X Y & \text { WE } \\ \text { DTn20 } & \text { SE } & X^{2} & \\ \text { TRan } & \text { WE } & X^{3} & \\ \text { SIDSpr } & \text { CTI } & Y^{2} & \\ \text { PET } & & Y^{3} & \\ \text { AET } & & X^{2} Y & \end{array}$

Final favourability model

\begin{tabular}{lrrlll}
\multicolumn{1}{c}{ Variables } & B & SE & Sig & Evaluation metrics & \\
(Intercept) & $2.55 \mathrm{E}+00$ & $3.27 \mathrm{E}+00$ & $\mathrm{~ns}$ & AUC & 0.9451618 \\
SIDSpr & $-1.18 \mathrm{E}+00$ & $3.93 \mathrm{E}-01$ & $* *$ & Kappa & 0.4641254 \\
PET & $-1.51 \mathrm{E}-02$ & $1.97 \mathrm{E}-03$ & $* * *$ & Sensitivity & 0.9259259 \\
Spatial & $4.74 \mathrm{E}-01$ & $8.02 \mathrm{E}-02$ & $* * *$ & Specificity & 0.8375391 \\
DAlt & $1.93 \mathrm{E}-03$ & $3.01 \mathrm{E}-04$ & $* * *$ & CCR & 0.8460814 \\
U100 & $-1.73 \mathrm{E}-02$ & $2.88 \mathrm{E}-03$ & $* * *$ & $\mathrm{HL}$ & $8.0409874 \mathrm{~ns}$ \\
Calc & $2.47 \mathrm{E}+00$ & $1.32 \mathrm{E}+00$ & $\mathrm{~ns}$ & & \\
DTn20 & $6.08 \mathrm{E}-02$ & $1.61 \mathrm{E}-02$ & $* * *$ & & \\
NIAL & $-1.42 \mathrm{E}+00$ & $4.84 \mathrm{E}-01$ & $* *$ & & \\
Clay & $4.07 \mathrm{E}+00$ & $1.32 \mathrm{E}+00$ & $* *$ & & \\
DP30Sum & $-3.26 \mathrm{E}-01$ & $1.50 \mathrm{E}-01$ & $*$ & & \\
TRan & $1.07 \mathrm{E}-01$ & $4.91 \mathrm{E}-02$ & $*$ & & \\
BLF & 1.1594021 & 0.4496624 & $* *$ & & \\
MF & 1.5278942 & 0.5935913 & $*$ & & \\
Shrub & 0.9421291 & 0.4291355 & $*$ & & \\
CTI & 0.2898229 & 0.1644539 & $\mathrm{~ns}$ & & \\
DHi & 0.0058643 & 0.0034182 & $\mathrm{~ns}$ & & \\
Sil & 3.4491757 & 1.3114712 & $* *$ & & \\
Grav & 3.4589674 & 1.3913402 & $*$ & &
\end{tabular}




\section{Canis lupus}

Pairwise correlations, spatial descriptor and FDR

Human, lithology and land use variables were not correlated, thus all of them were selected in pairwise correlations.

Variables selected

$\begin{array}{llll}\text { Climate } & \text { Topography } & \text { Spatial } & \text { Variables excluded by the FDR } \\ \text { DP30Win Alt } & Y & \text { Shrub } \\ \text { DP30Sum SE } & X & \text { CF } \\ \text { DTnOSum WE } & X Y & \text { DHi } \\ \text { DTn20 CTI } & X^{2} & \text { Clay } \\ \text { TRan } & X^{3} & \text { WE } \\ \text { TnJul } & Y^{2} & \\ \text { SIDWin } & Y^{3} & \\ \text { ROff } & X^{2} Y & \\ \text { AET } & X^{2} & \end{array}$

Final favourability model

\begin{tabular}{lrrlll}
$\begin{array}{l}\text { Variables } \\
\text { (Intercept) }\end{array}$ & \multicolumn{1}{c}{ B } & \multicolumn{1}{c}{ SE } & Sig & Evaluation metrics & \\
Spatial & $9.86 \mathrm{E}-01$ & $1.06 \mathrm{E}+00$ & $\mathrm{~ns}$ & AUC & 0.9549853 \\
TnJul & $-4.44 \mathrm{E}-01$ & $6.54 \mathrm{E}-02$ & $* * *$ & Kappa & 0.7057846 \\
HPd & $-3.51 \mathrm{E}-03$ & $1.06 \mathrm{E}-03$ & $* * *$ & Sensitivity & 0.9214418 \\
Past & $-1.91 \mathrm{E}+00$ & $7.94 \mathrm{E}-01$ & $*$ & CCR & 0.8833412 \\
AET & $1.42 \mathrm{E}-03$ & $6.67 \mathrm{E}-04$ & $*$ & $\mathrm{HL}$ & 0.8911078 \\
SIDWin & $1.52 \mathrm{E}+00$ & $4.82 \mathrm{E}-01$ & $* *$ & & $64.9676153 * * *$ \\
ROff & $5.48 \mathrm{E}-04$ & $2.49 \mathrm{E}-04$ & $*$ & & \\
DP30Sum & $2.88 \mathrm{E}-01$ & $1.77 \mathrm{E}-01$ & $\mathrm{~ns}$ & & \\
U500 & $1.87 \mathrm{E}-03$ & $1.04 \mathrm{E}-03$ & $\mathrm{~ns}$ & & \\
TRan & $1.37 \mathrm{E}-01$ & $4.17 \mathrm{E}-02$ & $* *$ & & \\
Alt & $-9.77 \mathrm{E}-04$ & $3.45 \mathrm{E}-04$ & $* *$ & & \\
DTn20 & 0.029119 & 0.0096646 & $* *$ & & \\
BLF & 0.997867 & 0.4280201 & $*$ & & \\
MF & 1.1523212 & 0.5657367 & & &
\end{tabular}




\section{Lutra lutra}

Pairwise correlations, spatial descriptor and FDR

Human, lithology and land use variables were not correlated, thus all of them were selected in pairwise correlations.

\section{Variables selected}

$\begin{array}{llll}\text { Climate } & \text { Topography } & \text { Spatial } & \text { Variables excluded by the FDR } \\ \text { DP1Win } & \text { Alt } & Y & \text { SE } \\ \text { DP30Spr } & \text { DAlt } & X & \\ \text { DP30Sum SE } & X Y & \\ \text { DTn0Sum WE } & X^{2} & \\ \text { DTn20 CTI } & Y^{2} & \\ \text { DTx25Spr } & Y^{3} & \\ \text { TRan } & X^{2} Y & \\ \text { TnSum } & X^{2} & \\ \text { SIDWin } & & \\ \text { AET } & \end{array}$

Final favourability model

\begin{tabular}{lrrlll} 
Variables & \multicolumn{1}{c}{ B } & SE & Sig & Evaluation metrics & \\
(Intercept) & $-4.69 \mathrm{E}+00$ & $1.50 \mathrm{E}+00$ & $* *$ & AUC & 0.8389843 \\
Spatial & $9.38 \mathrm{E}-01$ & $6.13 \mathrm{E}-02$ & $* * *$ & Kappa & 0.5040501 \\
NIAL & $-7.91 \mathrm{E}-01$ & $1.73 \mathrm{E}-01$ & $* * *$ & Sensitivity & 0.7481600 \\
U500 & $5.55 \mathrm{E}-03$ & $7.58 \mathrm{E}-04$ & $* * *$ & Specificity & 0.7654604 \\
AET & $3.43 \mathrm{E}-03$ & $3.94 \mathrm{E}-04$ & $* * *$ & CCR & 0.7552751 \\
TRan & $1.53 \mathrm{E}-01$ & $2.92 \mathrm{E}-02$ & $* * *$ & HL & $19.0502509 *$ \\
CF & $1.77 \mathrm{E}+00$ & $3.24 \mathrm{E}-01$ & $* * *$ & & \\
Shrub & $1.29 \mathrm{E}+00$ & $2.45 \mathrm{E}-01$ & $* * *$ & & \\
Grav & $9.60 \mathrm{E}-01$ & $2.19 \mathrm{E}-01$ & $* * *$ & & \\
DAlt & $1.43 \mathrm{E}-03$ & $2.07 \mathrm{E}-04$ & $* * *$ & & \\
DP30Spr & $-2.73 \mathrm{E}-01$ & $8.14 \mathrm{E}-02$ & $* * *$ & & \\
Alt & $-1.23 \mathrm{E}-03$ & $2.58 \mathrm{E}-04$ & $* * *$ & & \\
SIDWin & -1.1192614 & 0.243527 & $* * *$ & & \\
U100 & 0.0054707 & 0.0015113 & $* * *$ & & \\
Clay & 0.5139878 & 0.1405211 & $* * *$ & & \\
HPd & -0.0003003 & 0.0001439 & $*$ & & \\
BLF & 1.0727376 & 0.3473141 & $* *$ & & \\
DP30Sum & -0.3856548 & 0.1161857 & $* * *$ & & \\
DHi & 0.005279 & 0.0019783 & $* *$ & & \\
DP1Win & -0.0161865 & 0.0106114 & $\mathrm{~ns}$ & & \\
CTI & 0.1195982 & 0.0709648 & $\mathrm{~ns}$ & & \\
DTn20 & 0.012441 & 0.0048466 & $*$ & & \\
TnSum & -0.0866395 & 0.0424098 & $*$ & & \\
Gyps & 0.4284977 & 0.2949751 & ns & & \\
& & & & &
\end{tabular}




\section{Ursus arctos}

Pairwise correlations, spatial descriptor and FDR

Human, lithology and land use variables were not correlated, thus all of them were selected in pairwise correlations.

\section{Variables selected}

$\begin{array}{llll}\text { Climate } & \text { Topography } & \text { Spatial } & \text { Variables excluded by the FDR } \\ \text { DP30Spr Alt } & Y & \text { CF } \\ \text { DP30Sum SE } & X & \text { SE } \\ \text { DTnOSpr WE } & X Y & \text { WE } \\ \text { DTnOSum CTI } & X^{3} & \\ \text { DTn20 } & Y^{2} & \\ \text { TJul } & Y^{3} & \\ \text { TRan } & X^{2} Y & \\ \text { SIDSum } & X^{2} & \\ \text { ROff } & & \\ \text { AET } & & \end{array}$

Final favourability model

\begin{tabular}{lrrlll} 
Variables & \multicolumn{1}{c}{ B } & SE & Sig & Evaluation metrics \\
(Intercept) & $6.66 \mathrm{E}+00$ & $5.68 \mathrm{E}+00$ & $\mathrm{~ns}$ & AUC & 0.9909335 \\
Spatial & $7.20 \mathrm{E}-01$ & $8.85 \mathrm{E}-02$ & $* * *$ & Kappa & 0.5785035 \\
HPd & $-5.78 \mathrm{E}-02$ & $3.07 \mathrm{E}-02$ & $\mathrm{~ns}$ & Sensitivity & 0.9805825 \\
U500 & $1.41 \mathrm{E}-02$ & $2.29 \mathrm{E}-03$ & $* * *$ & Specificity & 0.9482556 \\
DHi & $3.12 \mathrm{E}-02$ & $6.82 \mathrm{E}-03$ & $* * *$ & CCR & 0.9495102 \\
Past & $6.75 \mathrm{E}+00$ & $1.58 \mathrm{E}+00$ & $* * *$ & $\mathrm{HL}$ & $10.6984415 \mathrm{~ns}$ \\
Clay & $1.45 \mathrm{E}+00$ & $6.28 \mathrm{E}-01$ & $*$ & & \\
ROff & $1.54 \mathrm{E}-03$ & $4.67 \mathrm{E}-04$ & $* * *$ & & \\
DTnOSum & $-9.84 \mathrm{E}-01$ & $3.25 \mathrm{E}-01$ & $* *$ & & \\
Grav & $3.26 \mathrm{E}+00$ & $1.29 \mathrm{E}+00$ & $*$ & & \\
Shrub & $3.54 \mathrm{E}+00$ & $9.30 \mathrm{E}-01$ & $* * *$ & & \\
DTn0Spr & $8.02 \mathrm{E}-02$ & $1.60 \mathrm{E}-02$ & $* * *$ & & \\
BLF & 2.2724366 & $0.9538473 *$ & & \\
MF & 3.6255715 & 1.2373222 & $* *$ & & \\
NIAL & 3.6905897 & 1.2625289 & $* *$ & & \\
CTI & -0.9455483 & 0.3461675 & $* *$ & &
\end{tabular}




\section{Felis silvestris}

Pairwise correlations, spatial descriptor and FDR

Human, lithology and land use variables were not correlated, thus all of them were selected in pairwise correlations.

\section{Variables selected}

$\begin{array}{llll}\text { Climate } & \text { Topography } & \text { Spatial } & \text { Variables excluded by the FDR } \\ \text { DP10Sum } & \text { Alt } & Y & \text { Sil } \\ \text { DTn0Sum } & \text { DAlt } & X & \text { U100 } \\ \text { DTn20 } & \text { SE } & X Y & \text { MF } \\ \text { PSpr } & \text { WE } & X^{2} & \text { TRan } \\ \text { TSpr } & \text { CTI } & X^{3} & \text { Gyps } \\ \text { TRan } & & Y^{2} & \text { U500 } \\ \text { AET } & & Y^{3} & \\ & & X^{2} Y & \\ & & X^{2} & \end{array}$

Final favourability model

\begin{tabular}{lrrlll} 
Variables & \multicolumn{1}{c}{ B } & SE & Sig & Evaluation metrics & \\
(Intercept) & $-1.19 \mathrm{E}+00$ & $3.54 \mathrm{E}-01$ & $* * *$ & AUC & 0.7683479 \\
DAlt & $1.25 \mathrm{E}-03$ & $1.46 \mathrm{E}-04$ & $* * *$ & Kappa & 0.3701999 \\
Spatial & $1.04 \mathrm{E}+00$ & $7.87 \mathrm{E}-02$ & $* * *$ & Sensitivity & 0.6823266 \\
Shrub & $1.42 \mathrm{E}+00$ & $2.04 \mathrm{E}-01$ & $* * *$ & Specificity & 0.7107955 \\
Alt & $6.03 \mathrm{E}-04$ & $1.24 \mathrm{E}-04$ & $* * *$ & CCR & 0.7012057 \\
BLF & $2.42 \mathrm{E}+00$ & $3.19 \mathrm{E}-01$ & $* * *$ & HL & $8.8091032 \mathrm{~ns}$ \\
PSpr & $-3.83 \mathrm{E}-03$ & $8.33 \mathrm{E}-04$ & $* * *$ & & \\
AET & $2.07 \mathrm{E}-03$ & $3.25 \mathrm{E}-04$ & $* * *$ & & \\
CF & $7.09 \mathrm{E}-01$ & $2.80 \mathrm{E}-01$ & $*$ & & \\
Past & $-1.84 \mathrm{E}+00$ & $7.17 \mathrm{E}-01$ & $*$ & & \\
NIAL & $-4.52 \mathrm{E}-01$ & $1.88 \mathrm{E}-01$ & $*$ & & \\
DTn20 & $-7.30 \mathrm{E}-03$ & $3.31 \mathrm{E}-03 *$ & & \\
DP10Sum & -0.0773781 & 0.0327702 & $*$ & & \\
Calc & 0.2352353 & $0.1088029 *$ & & \\
Grav & 0.3993633 & $0.1893693 *$ & & \\
HPd & -0.0002846 & 0.0001843 & $\mathrm{~ns}$ & & \\
WE & -0.0055291 & 0.0030949 & $\mathrm{~ns}$ & &
\end{tabular}




\section{Lynx pardinus}

Pairwise correlations, spatial descriptor and FDR

Human, lithology and land use variables were not correlated, thus all of them were selected in pairwise correlations.

Variables selected

$\begin{array}{llll}\text { Climate } & \text { Topography } & \text { Spatial } & \text { Variables excluded by the FDR } \\ \text { DP30Spr } & \text { Alt } & Y & \text { Gyps } \\ \text { DP30Sum } & \text { DAlt } & X & \text { Shrub } \\ \text { DTnOSpr } & \text { SE } & X Y & \text { Sil } \\ \text { DTnOSum } & \text { WE } & X^{2} & \text { TRan } \\ \text { DTn20 } & \text { CTI } & X^{3} & \text { Clay } \\ \text { DTx25Aut } & Y^{2} & \text { WE } \\ \text { TRan } & Y^{3} & \text { MF } \\ \text { SISSum } & X^{2} Y & \text { BLF } \\ \text { ROff } & X^{2} & \text { AET } \\ \text { AET } & & \text { HPd }\end{array}$

Final favourability model

$\begin{array}{lrrlll}\text { Variables } & \text { B } & \text { SE } & \text { Sig } & \text { Evaluation metrics } & \\ \text { (Intercept) } & -2.55 \mathrm{E}+02 & 7.29 \mathrm{E}+01 & * * * & \text { AUC } & 0.9886930 \\ \text { Spatial } & 3.50 \mathrm{E}-01 & 2.03 \mathrm{E}-01 & \mathrm{~ns} & \text { Kappa } & 0.1567660 \\ \text { DTx25Aut } & 2.74 \mathrm{E}-01 & 7.83 \mathrm{E}-02 & * * * & \text { Sensitivity } & 0.9655172 \\ \text { CF } & 6.14 \mathrm{E}+00 & 1.89 \mathrm{E}+00 & * * & \text { Specificity } & 0.9465808 \\ \text { NIAL } & -5.51 \mathrm{E}+00 & 2.21 \mathrm{E}+00 & * & \text { CCR } & 0.9466843 \\ \text { SISSum } & 3.12 \mathrm{E}+01 & 9.45 \mathrm{E}+00 & * * * & \mathrm{HL} & 9.6965038 \mathrm{~ns} \\ \text { DP30Sum } & 1.44 \mathrm{E}+01 & 3.70 \mathrm{E}+00 & * * * & & \\ \text { DP30Spr } & -2.28 \mathrm{E}+00 & 9.13 \mathrm{E}-01 & * & & \\ \text { ROff } & 1.02 \mathrm{E}-02 & 4.43 \mathrm{E}-03 & * & & \\ \text { Grav } & -1.84 \mathrm{E}+00 & 9.69 \mathrm{E}-01 & \mathrm{~ns} & & \end{array}$




\section{Cervus elaphus}

Pairwise correlations, spatial descriptor and FDR

Human, lithology and land use variables were not correlated, thus all of them were selected in pairwise correlations.

\section{Variables selected}

$\begin{array}{llll}\text { Climate } & \text { Topography } & \text { Spatial } & \text { Variables excluded by the FDR } \\ \text { DP10Spr } & \text { Alt } & Y & \text { Gyps } \\ \text { DP30Sum } & \text { DAlt } & X & \text { Calc } \\ \text { DTnOSum } & \text { SE } & X Y & \text { WE } \\ \text { DTn20Aut } & \text { WE } & X^{2} & \text { DP30Sum } \\ \text { TRan } & \text { CTI } & X^{3} & \text { MF } \\ \text { TnJan } & & Y^{2} & \\ \text { TXJul } & & Y^{3} & \\ \text { AET } & & X^{2} Y & \end{array}$

Final favourability model

\begin{tabular}{lrrlll}
\multicolumn{1}{c}{ Variables } & \multicolumn{1}{c}{ B } & SE & Sig & Evaluation metrics & \\
(Intercept) & $-9.88 \mathrm{E}+00$ & $7.68 \mathrm{E}-01$ & $* * *$ & AUC & 0.8060400 \\
U100 & $1.17 \mathrm{E}-02$ & $1.43 \mathrm{E}-03$ & $* * *$ & Kappa & 0.4235189 \\
Spatial & $7.20 \mathrm{E}-01$ & $7.44 \mathrm{E}-02$ & $* * *$ & Sensitivity & 0.7223899 \\
BLF & $3.40 \mathrm{E}+00$ & $3.14 \mathrm{E}-01$ & $* * *$ & Specificity & 0.7362366 \\
CF & $2.87 \mathrm{E}+00$ & $2.93 \mathrm{E}-01$ & $* * *$ & CCR & 0.7319141 \\
Shrub & $1.83 \mathrm{E}+00$ & $2.09 \mathrm{E}-01$ & $* * *$ & $\mathrm{HL}$ & $10.2530292 \mathrm{~ns}$ \\
TxJul & $1.38 \mathrm{E}-01$ & $2.25 \mathrm{E}-02$ & $* * *$ & & \\
DTn0Sum & $8.46 \mathrm{E}-01$ & $1.56 \mathrm{E}-01$ & $* * *$ & & \\
DP10Spr & $9.47 \mathrm{E}-02$ & $2.23 \mathrm{E}-02$ & $* * *$ & & \\
U500 & $3.92 \mathrm{E}-03$ & $7.44 \mathrm{E}-04$ & $* * *$ & & \\
SE & $8.49 \mathrm{E}-03$ & $2.85 \mathrm{E}-03$ & $* *$ & & \\
DAlt & $3.00 \mathrm{E}-04$ & $1.41 \mathrm{E}-04$ & $*$ & & \\
Sil & -0.2496909 & 0.1045836 & $*$ & & \\
AET & 0.0008928 & 0.0003652 & $*$ & & \\
HPd & 0.0001989 & 0.0001129 & $\mathrm{~ns}$ & & \\
TRan & 0.0692817 & 0.0337668 & $*$ & & \\
DTn20Aut & 0.0304919 & 0.0182856 & $\mathrm{~ns}$ & &
\end{tabular}




\section{Rupicapra pyrenaica}

Pairwise correlations, spatial descriptor and FDR

Human, lithology and land use variables were not correlated, thus all of them were selected in pairwise correlations.

Variables selected

$\begin{array}{llll}\text { Climate } & \text { Topography } & \text { Spatial } & \text { Variables excluded by the FDR } \\ \text { DP10Spr } & \text { Alt } & X & \text { Shrub } \\ \text { DP10Sum SE } & X Y & \text { Gyps } \\ \text { DTn0Sum } & \text { WE } & X^{2} & \text { Calc } \\ \text { DTn20 CTI } & X^{3} & \text { WE } \\ \text { Tann } & Y^{2} & \\ \text { TRan } & Y^{3} & \\ \text { AET } & X^{2} Y & \\ & & X Y^{2}\end{array}$

Final favourability model

$\begin{array}{lrclll}\text { Variables } & \text { B } & \text { SE } & \text { Sig } & \text { Evaluation metrics } & \\ \text { (Intercept) } & 2.86 \mathrm{E}+01 & 6.22 \mathrm{E}+00 & * * * & \text { AUC } & 0.9952173 \\ \text { Spatial } & 4.15 \mathrm{E}-01 & 8.37 \mathrm{E}-02 & * * * & \text { Kappa } & 0.6543732 \\ \text { Alt } & 3.05 \mathrm{E}-03 & 6.14 \mathrm{E}-04 & * * * & \text { Sensitivity } & 0.9770642 \\ \text { CTI } & -2.19 \mathrm{E}+00 & 3.67 \mathrm{E}-01 & * * * & \text { Specificity } & 0.9603143 \\ \text { U100 } & 2.08 \mathrm{E}-02 & 7.21 \mathrm{E}-03 & * * & \text { CCR } & 0.9610023 \\ \text { DHi } & 1.84 \mathrm{E}-02 & 9.35 \mathrm{E}-03 & * & \mathrm{HL} & 5.3400624 \mathrm{~ns} \\ \text { DP10Sum } & 2.03 \mathrm{E}-01 & 1.10 \mathrm{E}-01 & \mathrm{~ns} & & \\ \text { DTn0Sum } & 7.48 \mathrm{E}-01 & 4.18 \mathrm{E}-01 & \mathrm{~ns} & & \end{array}$




\section{Capra pyrenaica}

Pairwise correlations, spatial descriptor and FDR

Human, lithology and land use variables were not correlated, thus all of them were selected in pairwise correlations.

Variables selected

$\begin{array}{llll}\text { Climate } & \text { Topography } & \text { Spatial } & \text { Variables excluded by the FDR } \\ \text { DP01Win } & \text { Alt } & Y & \text { DTx25Spr } \\ \text { DP30Spr } & \text { DAlt } & X & \text { MF } \\ \text { DP30Sum } & \text { SE } & \text { XY } & \text { SE } \\ \text { DTnOSum WE } & X^{2} & \text { Clay } \\ \text { DTn20 CTI } & X^{3} & \text { AET } \\ \text { DTx25Spr } & Y^{2} & \text { DP30Sum } \\ \text { TRan } & Y^{3} & \\ \text { TnWin } & X^{2} Y & \\ \text { SISWin } & X^{2} & \\ \text { AET } & & \end{array}$

Final favourability model

$\begin{array}{lrrlll}\text { Variables } & \text { B } & \text { SE } & \text { Sig } & \text { Evaluation metrics } & \\ \text { (Intercept) } & 2.07 \mathrm{E}+00 & 2.72 \mathrm{E}+00 & \mathrm{~ns} & \text { AUC } & 0.9376692 \\ \text { Spatial } & 1.22 \mathrm{E}+00 & 5.74 \mathrm{E}-02 & * * * & \text { Kappa } & 0.5036426 \\ \text { CTI } & -4.28 \mathrm{E}-01 & 1.48 \mathrm{E}-01 & * * & \text { Sensitivity } & 0.9088099 \\ \text { DP30Spr } & 9.93 \mathrm{E}-01 & 1.17 \mathrm{E}-01 & * * * & \text { Specificity } & 0.8341558 \\ \text { DAlt } & 1.87 \mathrm{E}-03 & 2.91 \mathrm{E}-04 & * * * & \mathrm{CCR} & 0.843255 \\ \text { Shrub } & 1.86 \mathrm{E}+00 & 3.35 \mathrm{E}-01 & * * * & \mathrm{HL} & 7.4210190 \mathrm{~ns} \\ \text { TRan } & 2.57 \mathrm{E}-01 & 4.02 \mathrm{E}-02 & * * * & & \\ \text { DHi } & 1.68 \mathrm{E}-02 & 3.29 \mathrm{E}-03 & * * * & & \\ \text { CF } & 1.10 \mathrm{E}+00 & 4.36 \mathrm{E}-01 & * & & \\ \text { WE } & -1.24 \mathrm{E}-02 & 5.09 \mathrm{E}-03 * & * & \\ \text { Grav } & 8.07 \mathrm{E}-01 & 3.73 \mathrm{E}-01 & * & & \\ \text { NIAL } & -9.52 \mathrm{E}-01 & 4.06 \mathrm{E}-01 & & & \\ \text { DTn20 } & -0.0086115 & 0.004401 & \mathrm{~ns} & & \\ \text { Gyps } & -2.2376372 & 1.5361969 & \mathrm{~ns} & & \\ \text { U100 } & -0.0037727 & 0.0025533 & \mathrm{~ns} & & \end{array}$

